\title{
Análisis sistémico del sector de salud mediante la matriz de insumo-producto, 2000-2005
}

Fernando Salgueiro Perobelli, Mônica Viegas Andrade, Edson Paulo Domingues, Flaviane Sousa Santiago, Joilson de Assis Cabral y Lucas Barbosa Rodrigues

RESUMEN

En este trabajo se presenta un análisis sistémico del sector de salud en el Brasil, basado en el estudio de la estructura productiva sectorial y sus interacciones con los demás sectores de la economía. Se utilizan datos inéditos divulgados por el Instituto Brasileño de Geografía y Estadística (IBGE) sobre las Cuentas Nacionales de Salud y se propone una metodología de compatibilización del Sistema de Cuentas Nacionales (matriz de insumo-producto) con las Cuentas Satélite de Salud relativas a los años 2000 y 2005. Esa compatibilización permite entender las relaciones entre el sector de salud y los demás sectores de la economía por medio de indicadores de insumo-producto.

PALABRAS CLAVE

CLASIFICACIÓN JEL

AUTORES
Salud, servicios de salud, análisis de insumo-producto, economía de la salud, cuentas nacionales, Macroeconomía, productividad, Brasil

I10, D57

Fernando Salgueiro Perobelli es profesor asociado de la Universidad Federal de Juiz de Fora, Brasil. fernando.perobelli@uff.edu.br

Mônica Viegas Andrade es profesora asociada de la Universidad Federal de Minas Gerais, Brasil. mviegas@cedeplar.ufmg.br

Edson Paulo Domingues es profesor asociado de la Universidad Federal de Minas Gerais, Brasil. domingues.edson@gmail.com

Flaviane Sousa Santiago es asistente de investigación de la Universidad Federal de Minas Gerais, Brasil. santiago.flaviane@gmail.com

Joilson de Assis Cabral es profesor adjunto de la Universidad Federal Rural de Río de Janeiro, Brasil. cabraljoilson@gmail.com

Lucas Barbosa Rodrigues es maestro en Economía de la Universidad Federal de Juiz de Fora, Brasil. Lucasbr_rodrigues@hotmail.com 


\section{I}

\section{Introducción}

El sector de salud tiene importantes efectos en el nivel de bienestar social. Desde el punto de vista individual, el consumo de bienes y servicios de este sector afecta directamente al bienestar en la medida en que el estado de salud determina el grado de sentirse plenamente bien de los individuos. Desde el punto de vista macroeconómico, este factor incide en el crecimiento económico, pues compone el acervo de capital humano y determina la capacidad productiva de la economía (oms, 2001; Bloom, Canning y Sevilla, 2001). En los últimos años, los gastos en salud per cápita de las economías desarrolladas y en desarrollo han crecido en forma considerable (la tasa media de los países de la Organización para la Cooperación y el Desarrollo Económicos (OCDE) es del 3\% al año (OCDE, 2010). Ese aumento se explica en gran medida por la introducción de nuevas tecnologías y los cambios en el perfil epidemiológico de la población, dado que la mayor prevalencia de enfermedades crónicas determina un uso más intensivo de los servicios hospitalarios. En el Brasil los gastos en salud, públicos y privados, corresponden a alrededor del $8 \%$ del producto interno bruto (PIB). Esa cifra es similar a la media de los países de la OCDE, que en 2009 registraron un gasto medio del 8,9\% del PIB. En valores absolutos, el gasto brasileño, equivalente a 674 dólares per cápita a paridad de poder adquisitivo en 2006 (oms, 2009), es inferior a la media global de 790 dólares per cápita. Con respecto a la dinámica del sector en el Brasil, los gastos en salud como proporción del PIB se han mantenido casi constantes en los últimos años (IBGE, 2008). Esa relativa estabilidad se debe en parte al control presupuestario del gobierno, que frena los gastos públicos. En las economías con sistemas de salud predominantemente públicos es habitual que se realice un mayor control de los gastos en este ítem.

Además de la importancia del sector de salud en términos de participación en el producto, se pueden destacar otras características para entender su dinamismo y su interacción sectorial en la economía. La provisión de cuidados de salud, sobre todo en el caso de los servicios

$\square$ Los autores agradecen la financiación recibida para la realización de este trabajo por parte de la Coordinación de Perfeccionamiento del Personal de Nivel Superior (CAPES), en el ámbito del edicto del Programa Nacional de Cooperación Académica (PROCAD) del Consejo Nacional de Desarrollo Científico y Tecnológico (CNPq). de baja y media complejidad, es un proceso productivo intensivo en mano de obra, que se traduce en una elevada absorción de la población ocupada. La mayoría de los servicios no son comercializables y se caracterizan por una demanda vinculada a la localidad de residencia. Por otra parte, los cuidados de alta complejidad, en particular los servicios de diagnóstico y fabricación de medicamentos, se caracterizan por el uso intensivo de tecnología. En consecuencia, esos sectores de salud presentan una mayor interdependencia con el sector externo, ya sea directamente, por ejemplo a través de la adquisición de equipos, o indirectamente, por medio de insumos para la realización de procedimientos médicos. La intensidad del uso de la tecnología varía entre los países y parece estar relacionada con los mecanismos de financiación y prestación de esos servicios en cada país.

El objetivo de este artículo consiste en realizar un análisis sistémico del sector de salud en el Brasil, basado en el estudio de la estructura productiva y las interacciones con los demás sectores de la economía. Se utilizan datos inéditos divulgados por el Instituto Brasileño de Geografía y Estadística (IBGE) sobre las cuentas nacionales de salud y se propone una metodología de compatibilización del Sistema de Cuentas Nacionales (matriz de insumoproducto) con las Cuentas Satélite de Salud relativas a los años 2000 y $2005^{1}$. Esa compatibilización permite entender las relaciones entre este sector y los demás sectores de la economía y medir los efectos de las políticas sanitarias en los agregados macroeconómicos por medio de indicadores de insumo-producto. Además, el Sistema de Cuentas Nacionales de Salud adoptado en el Brasil sigue el modelo de cuentas internacional, que permite realizar comparaciones entre los países.

Las matrices de insumo-producto constituyen una herramienta apropiada para el análisis de la interdependencia de los sectores en una determinada economía. Su función principal es permitir la evaluación de los requisitos de producción de un sector necesarios para satisfacer una estructura sectorial de demanda final de bienes y servicios. La metodología de insumoproducto se ha aplicado a análisis en diferentes áreas.

\footnotetext{
1 En este trabajo se utilizan los cuadros de recursos y usos para los sectores de salud y los cuadros de usos de bienes y servicios para dichos sectores.
} 
En el Brasil existen aplicaciones relativas a gran parte de los sectores industriales -agrícola (Rodrigues y Guilhoto, 2004), transporte (Toyoshima y Ferreira, 2002; Betarelli Junior, Bastos y Perobelli, 2008), distribución del ingreso (Azzoni y otros, 2007; Moreira, 2007), energía (Perobelli, Mattos y Faria, 2007; Mattos y otros, 2008) y temas ambientales (Hilgemberg y Guilhoto, 2006; Imori y Guilhoto, 2007), entre otros-, pero son todavía escasos los estudios centrados específicamente en el sector de salud. La mayoría de las investigaciones a nivel internacional sobre dicho sector que utilizan la metodología de insumo-producto se refieren sobre todo a las relaciones intrasectoriales (Correa y Parker, 2005; Hongyi, 2009).

El presente artículo contribuye a la literatura sobre insumo-producto en el Brasil y a cubrir el vacío de análisis específicos del sector de salud. En un contexto de transición epidemiológica y envejecimiento de la población como el observado en el Brasil, es previsible que los gastos en salud tiendan a aumentar. Este incremento puede tener efectos diferenciados en la economía, según las relaciones del sector de salud con los demás sectores económicos. En ese sentido, la compatibilización de la matriz de insumo-producto brasileña constituye una herramienta importante para permitir un mayor nivel de desagregación del sector de salud. Además, en el marco de consolidación y expansión del sistema sanitario brasileño, en particular del sistema público, un mejor entendimiento de las relaciones intersectoriales es fundamental para optimizar la planificación de las políticas públicas de salud.

El documento se estructura de la siguiente manera a partir de esta Introducción. La sección II se refiere al sector de salud brasileño. En la sección III se aborda la compatibilización de las Cuentas Nacionales de Salud con el Sistema de Cuentas Nacionales. A su vez, en la sección IV se examinan las estadísticas descriptivas de la matriz de insumo-producto con apertura para la salud, en tanto que en la sección $V$ se lleva a cabo un análisis de los demás sectores de salud a partir del modelo de insumo-producto. Finalmente, en la sexta sección se ofrecen los resultados.

\section{II}

\section{El sector de salud en el Brasil}

En esta sección se describe el desempeño del sector de salud brasileño a partir de una muestra extendida de países de la OCDE, que incluye algunos países latinoamericanos. Se utilizan tres indicadores: gasto en el sector de salud como proporción del producto interno bruto (PIB), esperanza de vida al nacer y gasto en salud a paridad de poder adquisitivo. La esperanza de vida al nacer se emplea generalmente en la literatura como indicador del estado medio de salud de la población, ya que tiene en cuenta todos los grupos etarios y todas las cohortes sobrevivientes (Robine, Romieu y Cambois, 1999; Almeida y otros, 2000; Andrade, 2002; Camargo, Rodrigues y Machado, 2006; Terra y Queiroz, 2010). Si bien este indicador presenta algunas limitaciones, pues no incorpora la dimensión de morbilidad o calidad de vida de los individuos, constituye la medida más apropiada sobre todo para realizar comparaciones internacionales. Los indicadores de gasto como proporción del PIB y gasto a paridad de poder adquisitivo son complementarios. El primero se refiere a la asignación de los gastos en salud en los países, mientras que el segundo presenta una medida del nivel de gastos con relación a cada individuo.
Dos países pueden presentar la misma estructura de asignación de gastos en salud, pero con niveles de gastos muy dispares, de acuerdo con las diferencias en términos de ingresos per cápita.

En el gráfico 1 se presenta la relación entre la proporción del PIB gastada en salud y el PIB per cápita en 2007. El comportamiento de la curva muestra la relación monótona entre gastos en salud y PIB per cápita, pues en los países más ricos se asigna una proporción mayor del PIB al sector de salud. Si bien el PIB per cápita brasileño es bajo (7.185 dólares a paridad de poder adquisitivo), el país se sitúa en la curva, sugiriendo que la asignación de gastos al sector de salud en términos de participación relativa del producto sigue el mismo comportamiento que en los países desarrollados. Es interesante notar la situación del Brasil comparada con la de otros países latinoamericanos: si bien el PIB per cápita de México y Chile es similar al brasileño, el porcentaje asignado a la salud es muy inferior. Por otra parte, mientras que el PIB per cápita de la Argentina y Cuba es inferior al brasileño, la participación relativa de los gastos en salud es muy similar a la de los países desarrollados. Entre estos 
GRÁFICO 1

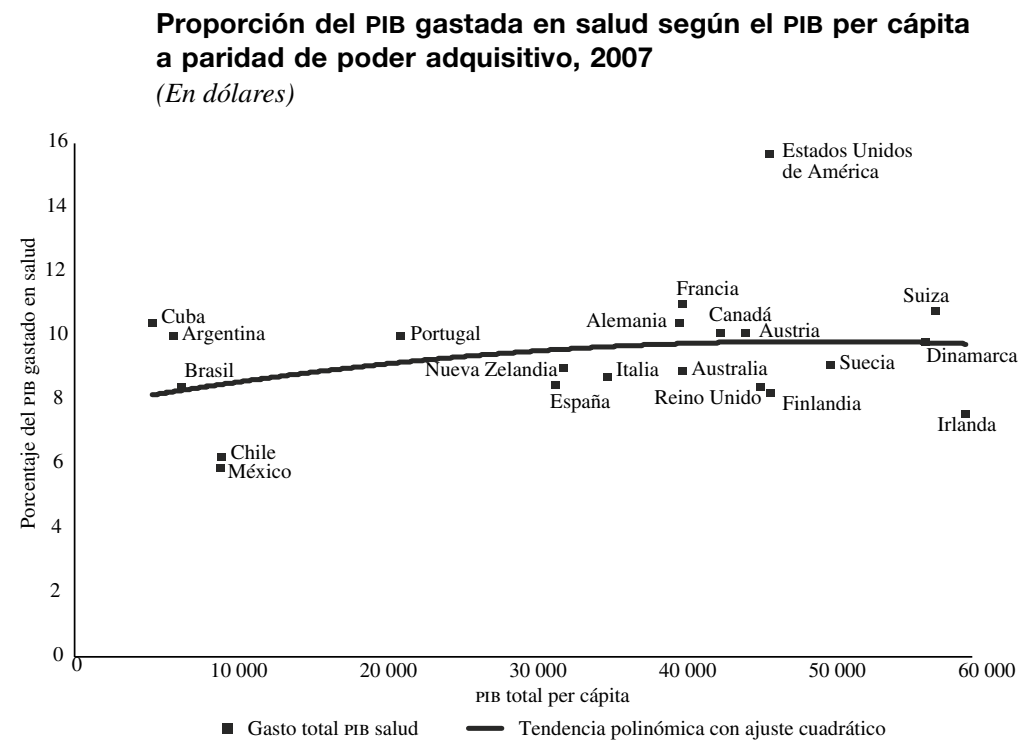

Fuente: Organización Mundial de la Salud (OMs), "Indicadores sanitarios mundiales", Estadísticas Sanitarias Mundiales 2010, Ginebra, 2010 [en línea] http://www.who.int/whosis/whostat/ES_WHS10_Full.pdf?ua=1; Banco Mundial, "PIB per cápita”, 2007 [en línea] http:// datos.bancomundial.org/indicador/NY.GDP.PCAP.CD.

últimos, los Estados Unidos de América registran el gasto más elevado, próximo al $16 \%$ del PIB, y presentan una posición muy por sobre la curva de ajuste entre los gastos en salud y el PIB per cápita. Otros países desarrollados, como Alemania $(10,4 \%)$, el Canadá $(10,1 \%)$ y Francia (11\%), están bien ajustados a la curva.

En el gráfico 2 se evidencia la relación entre la esperanza de vida al nacer y el PIB per cápita. La esperanza de vida es una medida de bienestar económico. El comportamiento de la curva muestra un formato cuadrático que sugiere una relación positiva, pero con tasas decrecientes de aumento de la esperanza de vida con respecto a los incrementos del PIB per cápita. En otras palabras, se sugiere un comportamiento de función de producción de salud cóncavo, que explicita que las ganancias de salud son mayores en los países más pobres. A medida que se expande el nivel de riqueza de la economía, los aumentos obtenidos en la salud de los individuos son cada vez menores, teniendo en cuenta la dimensión del tiempo de vida (mortalidad). La posición relativa del Brasil en ese gráfico es bastante desfavorable, pues se sitúa muy por debajo de la curva ajustada. Esa posición relativa sugiere que la esperanza de vida brasileña es muy inferior a la esperada en virtud de la riqueza del país. Ese comportamiento probablemente refleja el elevado nivel de mortalidad infantil del Brasil (cercano a 20 por cada 1.000 nacidos vivos) en comparación con los países desarrollados, cuyas tasas se sitúan en general por debajo de 10 por cada 1.000 nacidos vivos. De acuerdo con la literatura sobre economía de la salud, en el perfil epidemiológico brasileño coexisten enfermedades infectocontagiosas típicas de los países menos desarrollados, con enfermedades crónico-degenerativas típicas de los países desarrollados (Luna, 2002; Schramm y otros, 2004; Brasil, Ministerio de Salud, 2004 y 2010; Campelo, Gonçalves y Donadi, 2005).

Por último, en el gráfico 3 se corroboran los resultados del gráfico 2. En este caso se analiza el comportamiento de la esperanza de vida en relación con los gastos totales en salud calculados a paridad de poder adquisitivo. El formato de la curva es el mismo que se obtuvo anteriormente y refuerza el comportamiento cóncavo de la función de producción en salud. Un aspecto interesante de esa relación es que permite interpretar la posición relativa de los países en términos de eficiencia técnica, pues la curva muestra el nivel de esperanza de vida que cabría suponer en virtud del nivel de gastos en salud. La posición relativa del Brasil es bastante desfavorable y revela cierto grado de ineficiencia en los gastos (Marinho, Cardoso y Almeida, 2009; Ribeiro y Rodrigues Jr., 2006; Ribeiro, 2008). Según el parecer de los autores del presente artículo, esta obedece a la persistencia de problemas de salud típicos de países más pobres, derivados sobre todo de la falta de saneamiento 
GRÁFICO 2

Esperanza de vida al nacer según el PIB per cápita, 2007

(En años y dólares)

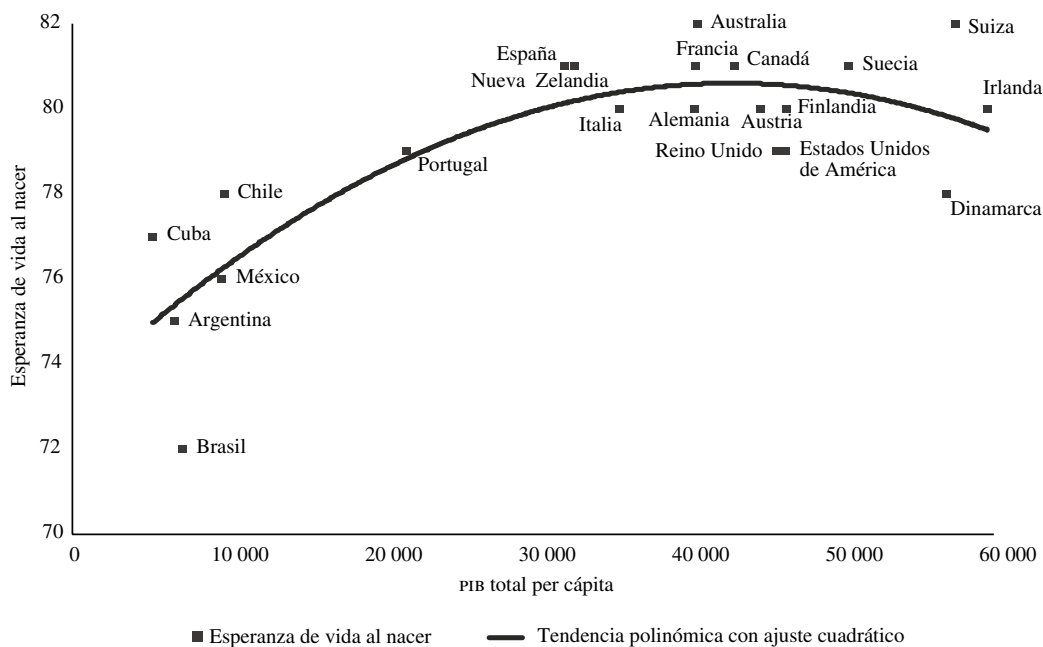

Fuente: Organización Mundial de la Salud (oms), "Indicadores sanitarios mundiales", Estadísticas Sanitarias Mundiales 2010, Ginebra, 2010 [en línea] http://www.who.int/whosis/whostat/ES_WHS10_Full.pdf?ua=1; Banco Mundial, "PIB per cápita", 2007 [en línea] http:// datos.bancomundial.org/indicador/NY.GDP.PCAP.CD.

GRÁFICO 3

Esperanza de vida al nacer según el gasto en salud per cápita a paridad de poder adquisitivo, 2007

(En años y dólares)

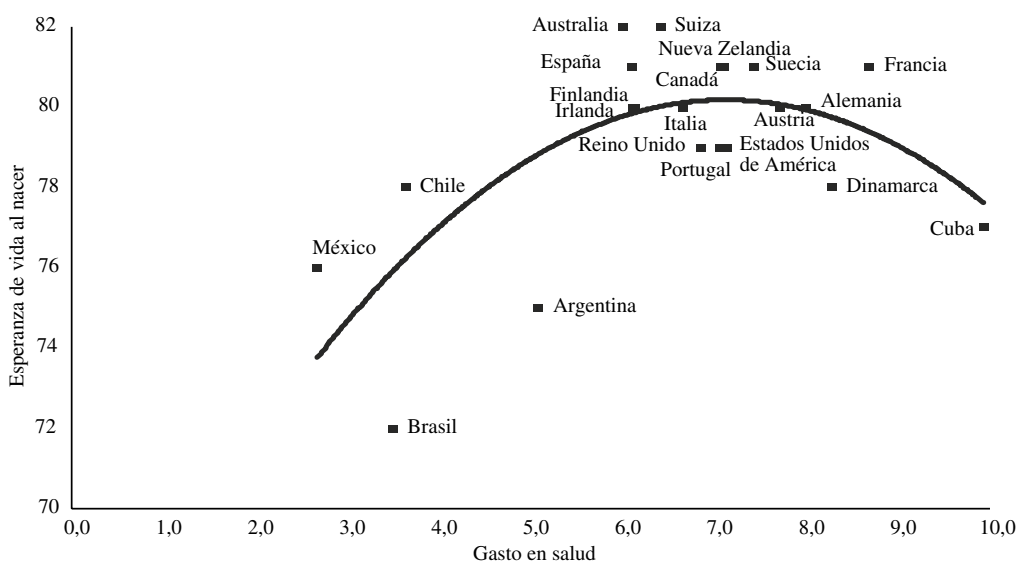

- Esperanza de vida al nacer _ Tendencia polinómica con ajuste cuadrático

Fuente: Organización Mundial de la Salud (oms), "Indicadores sanitarios mundiales", Estadísticas Sanitarias Mundiales 2010, Ginebra, 2010 [en línea] http://www.who.int/whosis/whostat/ES_WHS10_Full.pdf?ua=1; Banco Mundial, "PIB per cápita", 2007 [en línea] http:// datos.bancomundial.org/indicador/NY.GDP.PCAP.CD.

y servicios públicos básicos de salud para determinados segmentos de la población.

Los indicadores presentados ilustran la posición relativa del Brasil con respecto a los estándares mundiales de indicadores y gastos de salud. Para comprender mejor esa posición, en las próximas secciones se analizan las características estructurales del sector y la manera en que este se inserta en el sistema productivo. 


\section{III}

\section{Compatibilización de las Cuentas Nacionales de Salud con el Sistema de Cuentas Nacionales}

En esta sección se describe el procedimiento adoptado para la compatibilización de las matrices de insumoproducto del Brasil, con el objetivo de incorporar la desagregación de las actividades económicas del sector de salud en los años 2000 y 2005. Se utilizaron dos sistemas de datos: i) el Sistema de Cuentas Nacionales del IBGE (2000 y 2005), en el que se desagrega la matriz de insumo-producto brasileña en 55 sectores, y ii) el Sistema de Cuentas Nacionales de Salud, también divulgado por el IBGE, donde se presentan cinco actividades económicas del sector de salud que se suman a las ya existentes. La nueva versión de la matriz de insumo-producto propuesta en este trabajo comprende 60 sectores, que corresponden a los 55 sectores originales y los cinco sectores de la salud. Debido a que la estructura de las matrices de insumo-producto es similar con respecto a todos los años disponibles, se adopta el mismo procedimiento.

En los cuadros 1 y 2 se presentan la desagregación según la tecnología de la actividad y el producto en los Sistemas de Cuentas Nacionales (desagregación original) y la respectiva desagregación en el Sistema de Cuentas Nacionales de Salud. En la primera columna se detalla el subsector o producto original del Sistema de Cuentas Nacionales y en la segunda la clasificación disponible en el Sistema de Cuentas Nacionales de Salud propuesta por el IBGE. Como se puede apreciar, se crearon cuatro subsectores y siete productos adicionales relacionados con la salud.

Del Sistema de Cuentas Nacionales se utilizaron tres cuadros principales, a saber: el cuadro de recursos de bienes y servicios, que presenta la oferta de estos en la economía a precios corrientes y los valores de importación; el cuadro de usos de bienes y servicios a precios al consumidor, en que se proporciona el valor agregado de la economía, y el cuadro de oferta y demanda de la producción a precios básicos, en que se detallan los valores de consumo intermedio y de la demanda final. Los tres cuadros comprenden originalmente 110 productos y 55 sectores, configurando una matriz de 110x55.

Del Sistema de Cuentas Nacionales de Salud se utilizaron los datos relativos al valor total de la producción obtenidos en el cuadro de recursos de bienes y servicios, y los datos referidos al consumo intermedio y el valor agregado obtenidos en el cuadro de usos de bienes y servicios.

La aplicación de la metodología de insumo-producto requiere la definición del tipo de tecnología (sector o producto) bajo el cual se construirá la matriz. En este artículo se optó por un modelo de insumo-producto con tecnología basada en el sector, de modo que la matriz final es cuadrada 60x60 y se construye a partir de la matriz de cuota de mercado (market-share). Los procedimientos adoptados en la compatibilización se describen con mayor detalle en el anexo I.

CUADRO 1

\section{Descripción de los subsectores del sector de salud en los Sistemas} de Cuentas Nacionales y Cuentas Nacionales de Salud

\begin{tabular}{ll} 
Sector de salud & Demás sectores de salud \\
\hline $\begin{array}{l}\text { Productos farmacéuticos } \\
\text { Fabricación de aparatos para uso } \\
\text { médico-hospitalario y odontológico }\end{array}$ & Fabricación de productos farmacéuticos \\
Comercio & Fabricación de aparatos para uso médico-hospitalario y odontológico \\
& Otros comercios \\
Intermediación financiera y seguros & $\begin{array}{l}\text { Intermediación financiera y otros seguros } \\
\text { Asistencia médica complementaria }\end{array}$ \\
Salud mercantil & $\begin{array}{l}\text { Actividades de atención hospitalaria } \\
\text { Otras actividades relacionadas con la atención de la salud } \\
\text { Servicios sociales privados }\end{array}$ \\
\hline
\end{tabular}

Fuente: Instituto Brasileño de Geografía y Estadística (IBGE), Sistema de Contas Nacionais y Contas Nacionais em Saúde, 2005. 
CUADRO 2

Producto original y desagregación de los productos

en las Cuentas Nacionales de Salud

\begin{tabular}{ll}
\hline Producto de salud & Subproductos de salud \\
\hline Productos farmacéuticos & Productos farmoquímicos \\
& Medicamentos para uso humano \\
& Medicamentos para uso veterinario \\
& Materiales para uso médico, hospitalario y odontológico \\
Comercio & Otros comercios \\
& Comercio de productos farmacéuticos, médicos, ortopédicos y odontológicos \\
Intermediación financiera y seguros & Intermediación financiera y otros seguros \\
Salud mercantil & Plan de salud -incluido seguro de salud \\
& Actividades de atención hospitalaria \\
& Otras actividades relacionadas con la atención de la salud \\
& Servicios sociales privados
\end{tabular}

Fuente: Instituto Brasileño de Geografía y Estadística (IBGE), Sistema de Contas Nacionais y Contas Nacionais em Saúde, 2005.

\section{IV \\ Estadísticas descriptivas de la matriz de insumo-producto con apertura para la salud}

En el cuadro 3 se presenta la participación relativa de los demás sectores de salud en el PIB, el empleo y el valor bruto de la producción en los años 2000 y 2005 , respectivamente. El sector de salud en su conjunto representó cerca del 7\% del PIB y el $4 \%$ del empleo total generado en el país. Entre los demás subsectores se destacan la salud pública y otras actividades relacionadas con esta. La participación de este subsector en el PIB y el empleo se debe a la importancia del Sistema Único de Salud en la prestación y el financiamiento de los servicios de salud. Si bien la participación de los subsectores de fármacos y fabricación de aparatos e instrumentos médicos en la generación de empleo es bastante reducida, su participación en el PIB y el valor bruto de la producción es muy superior, lo que indica una elevada relación capital-producto.

CUADRO 3

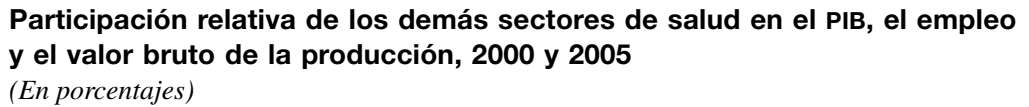

\begin{tabular}{|c|c|c|c|c|c|c|}
\hline \multirow{2}{*}{ Sectores } & \multicolumn{2}{|c|}{ PIB } & \multicolumn{2}{|c|}{ Empleo } & \multicolumn{2}{|c|}{$\begin{array}{l}\text { Valor bruto de } \\
\text { la producción }\end{array}$} \\
\hline & 2000 & 2005 & 2000 & 2005 & 2000 & 2005 \\
\hline Fabricación de productos farmacéuticos & 0,71 & 0,61 & 0,05 & 0,04 & 0,70 & 0,58 \\
\hline Aparatos e instrumentos médico-hospitalarios, de medición y ópticos & 0,32 & 0,33 & 0,12 & 0,13 & 0,29 & 0,28 \\
\hline Comercio de productos farmacéuticos, médicos, ortopédicos y odontológicos & 0,40 & 0,44 & 0,71 & 0,75 & 0,38 & 0,41 \\
\hline Asistencia médica complementaria & 0,26 & 0,19 & 0,06 & 0,07 & 0,33 & 0,22 \\
\hline Actividades de atención hospitalaria & 1,16 & 1,19 & 0,27 & 0,25 & 0,70 & 0,70 \\
\hline Otras actividades relacionadas con la atención de la salud & 1,89 & 1,57 & 1,04 & 1,14 & 1,15 & 0,92 \\
\hline Servicios sociales privados & 0,25 & 0,21 & 0,42 & 0,45 & 0,15 & 0,12 \\
\hline Salud pública & 2,47 & 2,73 & 1,37 & 1,40 & 1,45 & 1,55 \\
\hline Total & 7,46 & 7,27 & 4,04 & 4,23 & 5,16 & 4,79 \\
\hline
\end{tabular}

Fuente: Instituto Brasileño de Geografía y Estadística (IBGE), Sistema de Contas Nacionais y Contas Nacionais em Saúde, 2000 y 2005. 
Para verificar la estructura de eslabonamiento de los demás sectores de salud se presentan las estadísticas típicas del análisis de insumo-producto con respecto a los años 2000 y 2005 . En los gráficos 4 y 5 se aprecia la distribución de las ventas de los demás sectores de salud para consumo intermedio y componentes de la demanda final en 2000 y 2005 , respectivamente. A título comparativo, se incluye la composición de las ventas de grandes sectores agregados (agropecuario, extractivo, industria, comercio y servicios). Cabe notar que las familias representan una proporción muy baja de las ventas de salud pública, cuyo consumo corresponde básicamente al gobierno. En realidad, el consumo de salud pública de las familias se contabiliza en el consumo del gobierno, pues como bien público de libre acceso no está pagado (directamente) por las familias.

El análisis de los gráficos 4 y 5 revela dos modelos de relación intersectorial asociados a los demás sectores de salud. Un primer grupo de ellos, más orientado a la actividad industrial, está encabezado por la rama de asistencia médica complementaria, que registra la mayor participación en las ventas destinadas al consumo intermedio. De ese modo, una parte considerable de la producción de esos sectores se convierte en insumo de producción de otros sectores, generando potencialmente eslabonamientos ascendentes en la economía. Ese grupo está formado por los siguientes sectores de salud: fabricación de productos farmacéuticos, fabricación de aparatos para uso médico-hospitalario y odontológico, comercio de productos farmacéuticos, médicos, ortopédicos y odontológicos y asistencia médica complementaria. Este último está ligado al consumo del sector de la administración pública, que representa la parte financiada por el gobierno.

El segundo grupo de sectores comprende actividades relacionadas con la provisión de cuidados médicos y el principal destino de sus ventas es el consumo de las familias. En este grupo se incluyen los sectores de actividades de atención hospitalaria, otras actividades relacionadas con la atención de la salud y servicios sociales privados. Estas actividades suponen un escaso eslabonamiento ascendente, pues no son insumos representativos de otros rubros de la economía, sino que constituyen esencialmente servicios de consumo final. La mayoría de estas actividades corresponden a servicios de salud prestados en la localidad de residencia de los individuos. Este modelo de relación intersectorial se repite en los dos años analizados. La comparación de la estructura de ventas de estos sectores con la de los agregados sectoriales refuerza esa caracterización. La participación del primer grupo en el consumo intermedio es similar a la verificada para la industria y el comercio.

GRÁFICO 4

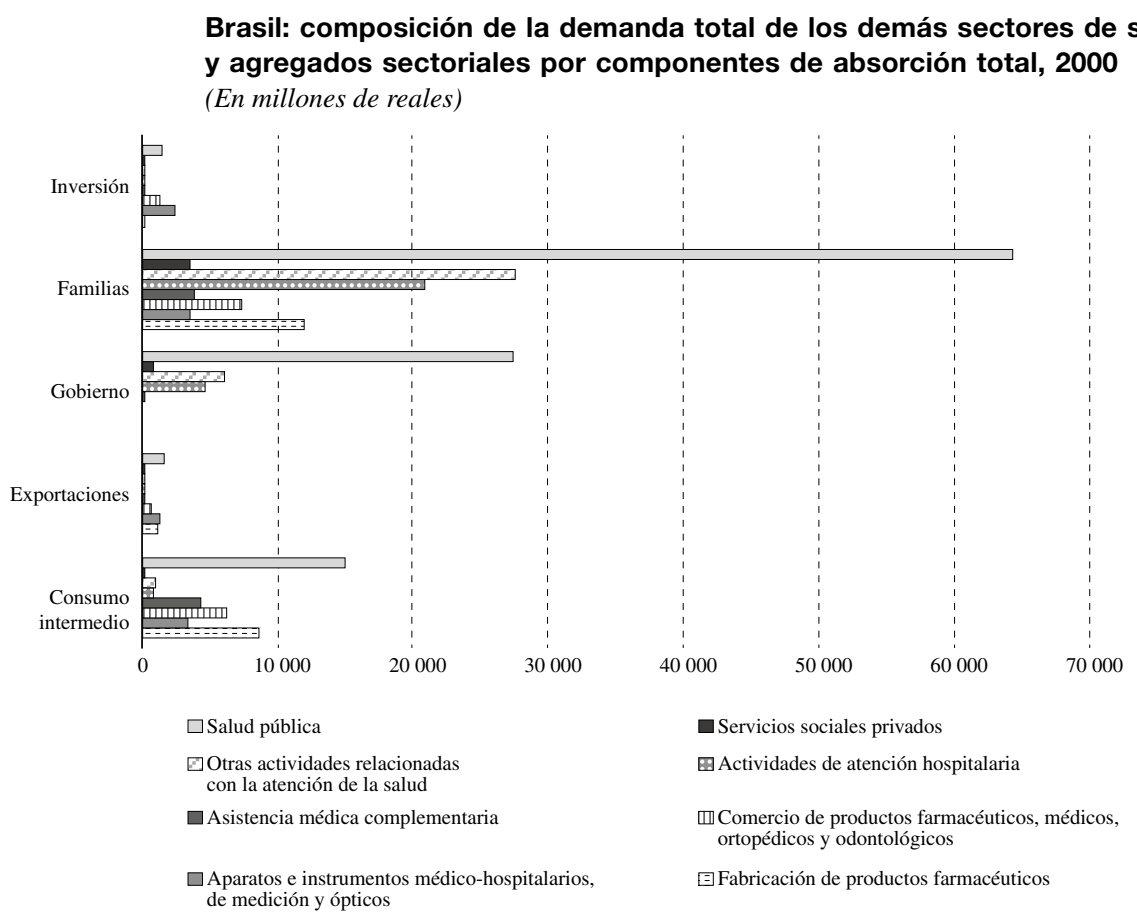

Fuente: Instituto Brasileño de Geografía y Estadística (IBGE), Sistema de Contas Nacionais y Contas Nacionais em Saúde, 2000. 
GRÁFICO 5

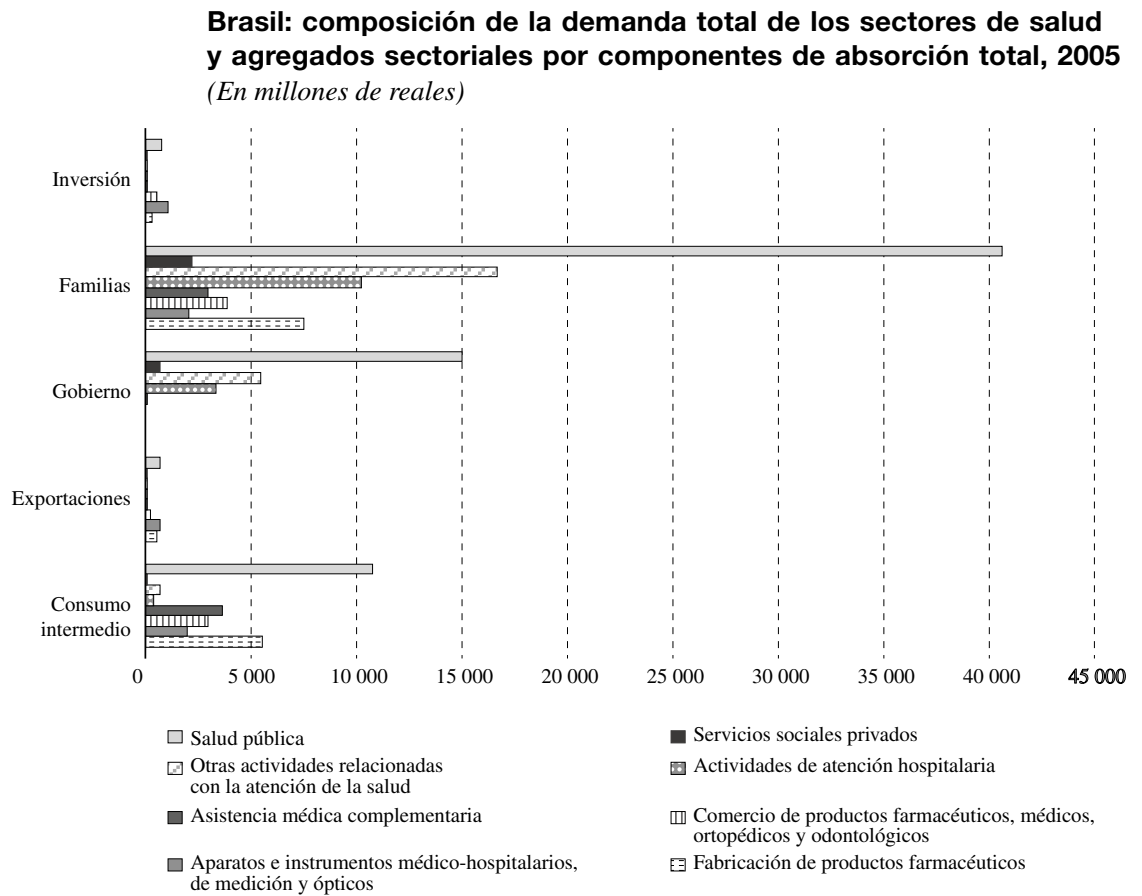

Fuente: Instituto Brasileño de Geografía y Estadística (IBGE), Sistema de Contas Nacionais y Contas Nacionais em Saúde, 2000.

Cabe también mencionar que el sector de salud brasileño está básicamente orientado a la demanda interna, de modo que el porcentaje de ventas destinadas a la exportación es bastante reducido.
En el cuadro 4 se presentan los datos relativos a la estructura de costo de los sectores de salud desagregados sobre la base de consumo intermedio nacional, valor agregado e importación, en 2000 y 2005 . Llama la

CUADRO 4

Brasil: composición relativa de las compras de los sectores de salud y agregados sectoriales, 2000 y 2005

(En porcentajes)

\begin{tabular}{|c|c|c|c|c|c|c|}
\hline \multirow[b]{2}{*}{ Sectores } & \multicolumn{3}{|c|}{2000} & \multicolumn{3}{|c|}{2005} \\
\hline & $\begin{array}{l}\text { Consumo } \\
\text { intermedio }\end{array}$ & Importación & $\begin{array}{c}\text { Valor } \\
\text { agregado }\end{array}$ & $\begin{array}{c}\text { Consumo } \\
\text { intermedio }\end{array}$ & Importación & $\begin{array}{c}\text { Valor } \\
\text { agregado }\end{array}$ \\
\hline Fabricación de fármacos & 41,4 & 15,9 & 42,7 & 43 & 14,6 & 42,3 \\
\hline Fabricación de aparatos & 20,1 & 10,4 & 69,5 & 21,8 & 11,7 & 66,5 \\
\hline Comercio salud & 30,8 & 0 & 69,2 & 30,5 & 0 & 69,5 \\
\hline Asistencia complementaria & 49,7 & 0 & 50,3 & 49,9 & 0 & 50,1 \\
\hline Actividades de atención hospitalaria & 49,7 & 8,1 & 42,2 & 50 & 9,4 & 40,6 \\
\hline Otras actividades relacionadas con la salud & 30,8 & 5 & 64,2 & 36,1 & 6,8 & 57,1 \\
\hline Servicios sociales privados & 44,4 & 7,2 & 48,4 & 39,5 & 7,5 & 53 \\
\hline Salud pública & 32,5 & 6,7 & 60,8 & 37 & 7,8 & 55,2 \\
\hline Agropecuario & 35,6 & 4,7 & 59,8 & 41,1 & 4,8 & 54,1 \\
\hline Extractivo & 45,2 & 8,5 & 46,3 & 48,9 & 8,7 & 42,4 \\
\hline Industria & 55,6 & 12,1 & 32,2 & 57,6 & 12,1 & 30 \\
\hline Comercio & 24,8 & 5,1 & 70,1 & 24,9 & 5,2 & 69,9 \\
\hline
\end{tabular}

Fuente: Instituto Brasileño de Geografía y Estadística (IBGE), Sistema de Contas Nacionais y Contas Nacionais em Saúde, 2000. 
atención el peso de las importaciones en los costos de los sectores de salud que desarrollan actividades industriales, es decir, la fabricación de productos farmacéuticos y la fabricación de aparatos e instrumentos para uso médicohospitalario y odontológico. Esa participación es similar a la observada con respecto a la industria en los dos años analizados. Por otra parte, la participación de esos dos sectores en el consumo intermedio es inferior a la media de la industria, lo que sugiere escasos efectos de eslabonamiento regresivo en la estructura productiva. Además, la expansión de estos sectores acarrea un aumento de la demanda de importaciones, lo que puede repercutir en forma significativa en la balanza comercial del país. El sector de actividades de atención hospitalaria es el que registra la composición de costos más próxima a la de la industria. Esto probablemente refleja la complejidad de los servicios prestados, que son intensivos tanto en equipos como en diversos servicios nacionales y necesitan relativamente menos importaciones. La composición de costos de los demás sectores de salud es similar a la del sector de servicios en general, con mayor participación del valor agregado y menor porcentaje de importaciones.

Los indicadores analizados en esta sección mostraron algunas características de los sectores de salud en su interrelación directa con los demás sectores de la economía, en términos de compras, ventas y costos. Se puede realizar un análisis complementario a partir del modelo de insumo-producto resultante de la matriz construida con los nuevos sectores de salud.

\section{V}

\section{Análisis de los sectores de salud a partir del modelo de insumo-producto}

El análisis de multiplicadores es un enfoque tradicional derivado del modelo de insumo-producto. Los multiplicadores complementan el análisis de la importancia de determinado sector en la economía, permitiendo evaluar los efectos directos e indirectos en el sistema económico derivados de perturbaciones exógenas, especialmente de la demanda final (Miller y Blair, 2009). En este trabajo se utilizan indicadores habituales de insumo-producto: multiplicadores de producción y empleo, índices de eslabonamiento regresivo y progresivo y sector clave. La construcción de estos indicadores sigue la metodología presentada en Miller y Blair (2009).

En síntesis, los multiplicadores de producción captan el efecto en la producción de la economía debido al incremento de una unidad monetaria de la demanda final del sector. De este modo, como el aumento de la demanda final de la producción del sector supone una necesidad de insumos directos, y esos insumos necesitan de más insumos, la necesidad indirecta de producción se vuelve indispensable para la satisfacción de esa demanda. Los coeficientes totales de la matriz inversa de Leontief captan ese efecto en todos los sectores, mientras que la suma a lo largo de las líneas mide el efecto multiplicador total de un sector. De la misma forma, tomando el empleo como coeficiente fijo con respecto a la producción de los sectores, los efectos multiplicadores de producción se reflejarán en efectos multiplicadores de empleo. Por último, el índice de eslabonamiento regresivo y progresivo y el sector clave son formulaciones basadas en los multiplicadores, con las que se procura identificar a los sectores que contribuyen por sobre la media de la economía en términos de efectos multiplicadores en la compra de insumos (regresivos) y en la venta de su producción (progresivos). En el anexo II se presenta la formalización de estos indicadores.

Un enfoque complementario al concepto de sector clave e índices de eslabonamiento es el del campo de influencia (Sonis y Hewings, 1992).

El concepto de campo de influencia se relaciona directamente con los cambios en los coeficientes técnicos de insumo-producto. Se define así:

$A=\left\|a_{i j}\right\|$ es la matriz de coeficientes directos;

$E=\left\|e_{i j}\right\|$ es la matriz de perturbaciones;

$B=(I-A)^{-1}=\left\|b_{i j}\right\|$ es la matriz inversa de Leontief antes de los efectos de las perturbaciones;

$B(\varepsilon)=(I-A-E)^{-1}=\left\|b_{i j}(\varepsilon)\right\|$ es la matriz inversa de Leontief después de los efectos de las perturbaciones.

Se supone una pequeña variación $\varepsilon$, en apenas un parámetro, $a_{i j}$ de la matriz $A$. Por ejemplo, se establece $\mathrm{i}=1$ y $\mathrm{j}=1$, de modo que: 


$$
E_{i j}=\left\{\begin{array}{ll}
\varepsilon, \text { se } & i=1, j=1 \\
0, \text { se } & i \neq 1, j \neq 1
\end{array}\right\}
$$

Según Sonis y Hewings (1992), el campo de influencia deriva de la siguiente relación:

$$
F\left(\varepsilon_{i j}\right)=\frac{\left\lfloor B\left(\varepsilon i_{j}\right)-B\right\rfloor}{\varepsilon_{i j}}
$$

donde $F\left(\varepsilon_{i j}\right)$ es la matriz de campo de influencia del cambio en el coeficiente de insumo-producto $a_{i j}$. De acuerdo con Guilhoto (2004), para determinar los

\section{VI}

\section{Resultados}

En esta sección se presentan los resultados obtenidos mediante el análisis de eslabonamientos de los sectores de salud en el contexto de la economía brasileña, utilizando un modelo de insumo-producto y sus indicadores habituales: i) multiplicadores simples de producción y empleo; ii) índices de eslabonamiento; iii) sector clave, $\mathrm{y}$ iv) campo de influencia.

Los índices de eslabonamiento progresivo y regresivo permiten evaluar la interacción entre los sectores, tanto con respecto a la oferta como a la demanda de insumos intermedios. De esta forma, se consideran sectores clave aquellos que poseen eslabonamientos por sobre la media tanto en la compra como en la venta de su producción al resto de la economía nacional.

En el anexo III se presentan los resultados de los índices de eslabonamiento progresivo y regresivo y la identificación de los sectores clave. Esos resultados se ilustran en los gráficos 6 y 7, en que se relacionan los índices de eslabonamiento progresivo y regresivo con la especificación de los sectores clave en 2000 y 2005. El eje horizontal corresponde al índice de eslabonamiento progresivo de cada sector y el eje vertical señala el índice de eslabonamiento regresivo.

En el cuadrante I de los gráficos 6 y 7 se encuentran los sectores clave, es decir, aquellos con eslabonamientos de compra y venta de insumos por sobre la media del resto de la economía. En el cuadrante II se hallan los sectores que poseen eslabonamientos progresivos por sobre la media y eslabonamientos regresivos por debajo coeficientes que presentarían los mayores campos de influencia sería necesario asociar a cada matriz $F\left(\varepsilon_{i j}\right)$ un valor igual a:

$$
S_{i j}=\sum_{k=1}^{n} \sum_{l=1}^{n}\left[f_{k l}\left(\varepsilon_{i j}\right)\right]^{2}
$$

De este modo, a partir del valor $S_{i j}$ se puede desarrollar una jerarquía de los coeficientes directos basados en los respectivos campos de influencia. En otras palabras, se pueden observar las relaciones sectoriales en términos de importancia en el efecto multiplicador en la economía. de la media, o sea, aunque la venta de sus productos en valores monetarios sea superior a la media del resto de la economía, la compra de insumos es inferior a ese valor. En el cuadrante III se muestran los sectores que tienen un bajo nivel de eslabonamiento de la producción, con pocas relaciones intersectoriales tanto en la venta como en la compra de productos en el mercado interno. Por último, si bien los sectores del cuadrante IV compran insumos intermedios nacionales por sobre la media, son poco utilizados como insumos de producción.

De acuerdo con los resultados, ninguno de los sectores de salud constituye un sector clave de la economía, tanto en el año 2000 (véase el gráfico 6) como en 2005 (véase el gráfico 7). Se trata de un resultado esperado porque, en general, los sectores de servicios poseen pocos eslabonamientos regresivos y progresivos, están más ligados a la demanda final (consumo de las familias y del gobierno) y presentan un uso intensivo de insumos importados (sector de fármacos).

Los sectores clave identificados para la economía brasileña fueron: i) alimentos y bebidas; ii) textil; iii) celulosa y productos de papel; iv) refinación de petróleo; v) productos químicos; vi) fabricación de resinas y elastómeros; vii) artículos de caucho y plástico; viii) fabricación de acero y derivados, y ix) productos de metal, con excepción de maquinaria y equipos. Se puede observar que no hubo variaciones en la lista de sectores clave entre los dos períodos. 
GRÁFICO 6

Dispersión de los índices de eslabonamiento regresivo y progresivo, 2000

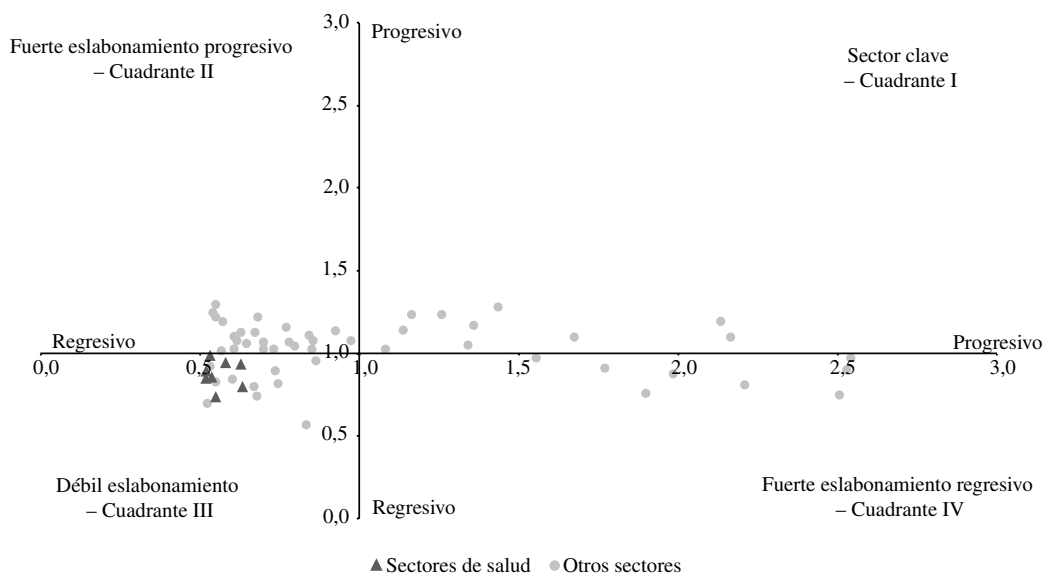

Fuente: elaboración propia.

GRÁFICO 7

Dispersión de los índices de eslabonamiento regresivo y progresivo, 2005

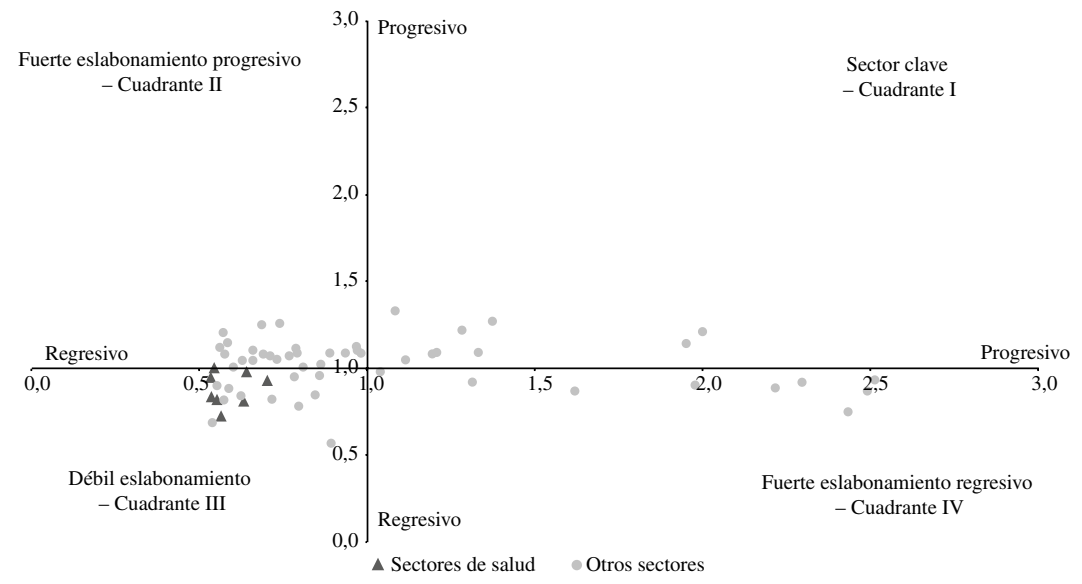

Fuente: elaboración propia.

Una observación más detallada de los multiplicadores de los sectores de salud permite deducir su efecto inferior a la media de la economía. El multiplicador de producción corresponde a una variación directa e indirecta de la producción total de la economía de todos los sectores, derivada de la variación exógena de una unidad monetaria de la demanda final de un determinado sector. Debido a que una parte considerable de las ventas de los sectores corresponde a la demanda final y sus coeficientes de compra intermedia son pequeños, se espera una colocación inferior de estos sectores en términos de eslabonamientos.
En el anexo IV se presentan los resultados de los multiplicadores relativos al Brasil en el período analizado (2000 y 2005). Dichos resultados son similares en los dos años, pues la estructura de insumo-producto presenta poca variación a lo largo del tiempo. Los multiplicadores simples de producción de los sectores de salud (véase el cuadro 5) presentaron valores por debajo de la media (1,88 en 2000 y 1,92 en 2005) en comparación con los demás sectores de la economía (véase el anexo IV). Cabe destacar que los sectores de servicios en general tienen pocos eslabonamientos ascendentes, pues sus ventas se destinan sobre todo a 


\begin{tabular}{|c|c|c|c|c|}
\hline \multirow{2}{*}{ Sectores de salud } & \multicolumn{4}{|c|}{ Multiplicador de la producción } \\
\hline & 2000 & Clasificación & 2005 & Clasificación \\
\hline Fabricación de fármacos & 1,75 & 39 & 1,79 & 40 \\
\hline Fabricación de aparatos & 1,36 & 58 & 1,40 & 58 \\
\hline Comercio de servicios de salud & 1,51 & 55 & 1,53 & 54 \\
\hline Asistencia complementaria & 1,83 & 35 & 1,80 & 39 \\
\hline Actividades de atención hospitalaria & 1,87 & 33 & 1,89 & 35 \\
\hline Otras actividades relacionadas con la salud & 1,54 & 53 & 1,64 & 47 \\
\hline Servicios sociales privados & 1,78 & 38 & 1,70 & 45 \\
\hline Salud pública & 1,57 & 51 & 1,62 & 48 \\
\hline Media general & \multicolumn{2}{|c|}{1,88} & \multicolumn{2}{|c|}{1,92} \\
\hline
\end{tabular}

Fuente: Instituto Brasileño de Geografía y Estadística (IBGE), Sistema de Contas Nacionais y Contas Nacionais em Saúde, 2000.

la demanda final. Asimismo, como se explicitó en la sección III, los elevados coeficientes de importación suponen menos efectos multiplicadores en la economía nacional, lo que caracteriza, por ejemplo, a los sectores de fabricación de fármacos y de actividades de atención hospitalaria. Entre los sectores de salud, el relativo a actividades de atención hospitalaria registró el mayor multiplicador de producción en 2000 y 2005: 1,87 y 1,88 , respectivamente (posiciones 33 y 35 en la clasificación de efecto multiplicador en la economía). En este caso, el aumento de 1 real en la producción del sector de actividades de atención hospitalaria genera un incremento de 1,89 reales en la demanda final de la economía.

Asociados con los multiplicadores de producción, los multiplicadores de empleo indican el número de puestos de trabajo generados en la economía por cada puesto creado en el sector de interés, incorporando los efectos directos e indirectos derivados de una variación en la demanda final.
En el cuadro 6 se presentan los multiplicadores de empleo de los sectores de salud. Un aumento de 1millón de reales en la demanda final produce una media de 59 empleos en 2000 y de 38 en 2005. Es interesante destacar que los multiplicadores de empleo se reducen en todos los sectores entre 2000 y 2005 , tal vez como consecuencia de aumentos de productividad en el período (véase el anexo IV). En los dos años analizados, los multiplicadores de los sectores de servicios sociales privados y comercio de servicios de salud se situaron por sobre la media nacional. Cabe resaltar el resultado del sector de servicios sociales privados, que ocupa el cuarto lugar como mayor multiplicador de empleo sectorial, con 103 empleos creados en la economía a raíz de la variación de 1 millón de reales en la demanda final en 2005. Esto se debe a la elevada participación directa de mano de obra en el sector. Los sectores de comercio de servicios de salud y otras actividades relacionadas con la salud también generaron empleos por sobre la media de la economía brasileña en 2005 (véase el cuadro 6).

CUADRO 6

Multiplicadores de empleo, 2000 y 2005

\begin{tabular}{lcccc} 
& \multicolumn{3}{c}{ Multiplicador del empleo } \\
\cline { 2 - 5 } Sectores de salud & 2000 & Clasificación & 2005 & Clasificación \\
\hline Fabricación de fármacos & 26 & 52 & 17 & 49 \\
Fabricación de aparatos & 34 & 39 & 20 & 41 \\
Comercio de servicios de salud & 89 & 13 & 54 & 15 \\
Asistencia complementaria & 30 & 44 & 22 & 36 \\
Actividades de atención hospitalaria & 44 & 27 & 43 & 28 \\
Otras actividades relacionadas con la salud & 54 & 24 & 103 & 4 \\
Servicios sociales privados & 133 & 4 & 36 & 23 \\
Salud pública & 58 & 22 & & 38
\end{tabular}

Fuente: Instituto Brasileño de Geografía y Estadística (IBGE), Sistema de Contas Nacionais y Contas Nacionais em Saúde, 2000. 
El campo de influencia, de acuerdo con lo calculado en la ecuación (3), delimita la importancia de cada una de las relaciones de compra y venta (insumo-producto) intersectoriales e intrasectoriales. En el cuadro 7 se presenta el campo de influencia del modelo de insumoproducto correspondiente al año 2005. Para facilitar la visualización, los resultados relativos a cada eslabón productivo se colocaron en escalas de grises que representan campos de influencia por sobre la media, es decir, los eslabones de mayor importancia para la economía en general. Esos eslabones se clasificaron en cuatro grupos, a saber: i) grupo A: eslabonamientos que presentaron una desviación estándar por sobre la media (color gris claro); ii) grupo B: entre 1 y 2 desviaciones por sobre la media (color gris medio); iii) grupo C: entre 2 y 3 desviaciones por sobre la media (color gris oscuro), y iv) grupo D: más de 3 desviaciones por sobre la media (color más oscuro). Siguiendo la estructura de insumo-producto, las líneas corresponden a los sectores que ofrecen insumos productivos, mientras que las columnas representan el destino de esos insumos. Las líneas y columnas indicadas con los números 18 y 19 se refieren a los sectores de salud.

Los resultados muestran pocos eslabones relevantes en los sectores de salud, en comparación con otros sectores. Específicamente, cabe señalar los resultados de 2005 relativos a los sectores "fabricación de productos farmacéuticos (18)", "fabricación de aparatos para uso médico-hospitalario y odontológico (19)" y "asistencia médica complementaria (48)". Los resultados del campo de influencia del año 2000 repiten ese patrón (véase el anexo V). Se verifica que el sector "fabricación de productos farmacéuticos (18)" tiene estrechos vínculos con sectores de otros ramos de la economía, entre ellos, los sectores de alimentos y bebidas (6), textil (8), celulosa y productos de papel (12), refinación de petróleo y coque (14) y electricidad y gas, agua, alcantarillado y limpieza urbana (41). Así, el principal eslabonamiento ascendente de los sectores de salud en la economía tiene lugar mediante las compras del sector de fármacos. Con respecto a la interdependencia intrasectorial, se destaca su eslabonamiento con el sector "asistencia médica complementaria (48)".

En lo que se refiere a la intensidad de los eslabonamientos de los sectores de salud es posible afirmar que:

i) El sector "fabricación de productos farmacéuticos (18)" presenta 7 eslabonamientos por sobre la media con respecto a las compras sectoriales y 12 eslabonamientos por sobre la media con relación a las ventas sectoriales.

ii) El sector "fabricación de aparatos para uso médico-hospitalario y odontológico" presenta 7 eslabonamientos por sobre la media con respecto a las compras sectoriales y 10 eslabonamientos por sobre la media con relación a las ventas sectoriales.

iii) El sector "asistencia médica complementaria (48)" presenta 7 eslabonamientos por sobre la media con respecto a las compras sectoriales y 14 eslabonamientos por sobre la media en relación con las ventas sectoriales.

iv) La intensidad de los eslabonamientos intrasectoriales (dentro del grupo de sectores que pertenecen al sector de salud) no es muy fuerte, tanto desde el punto de vista de las ventas como de las compras. Ellas están representadas por las líneas 44, 48, $54,55,56$ y 59 , que indican eslabonamientos con una desviación inferior a la media. En algunos casos los eslabonamientos están por debajo de este patrón. 


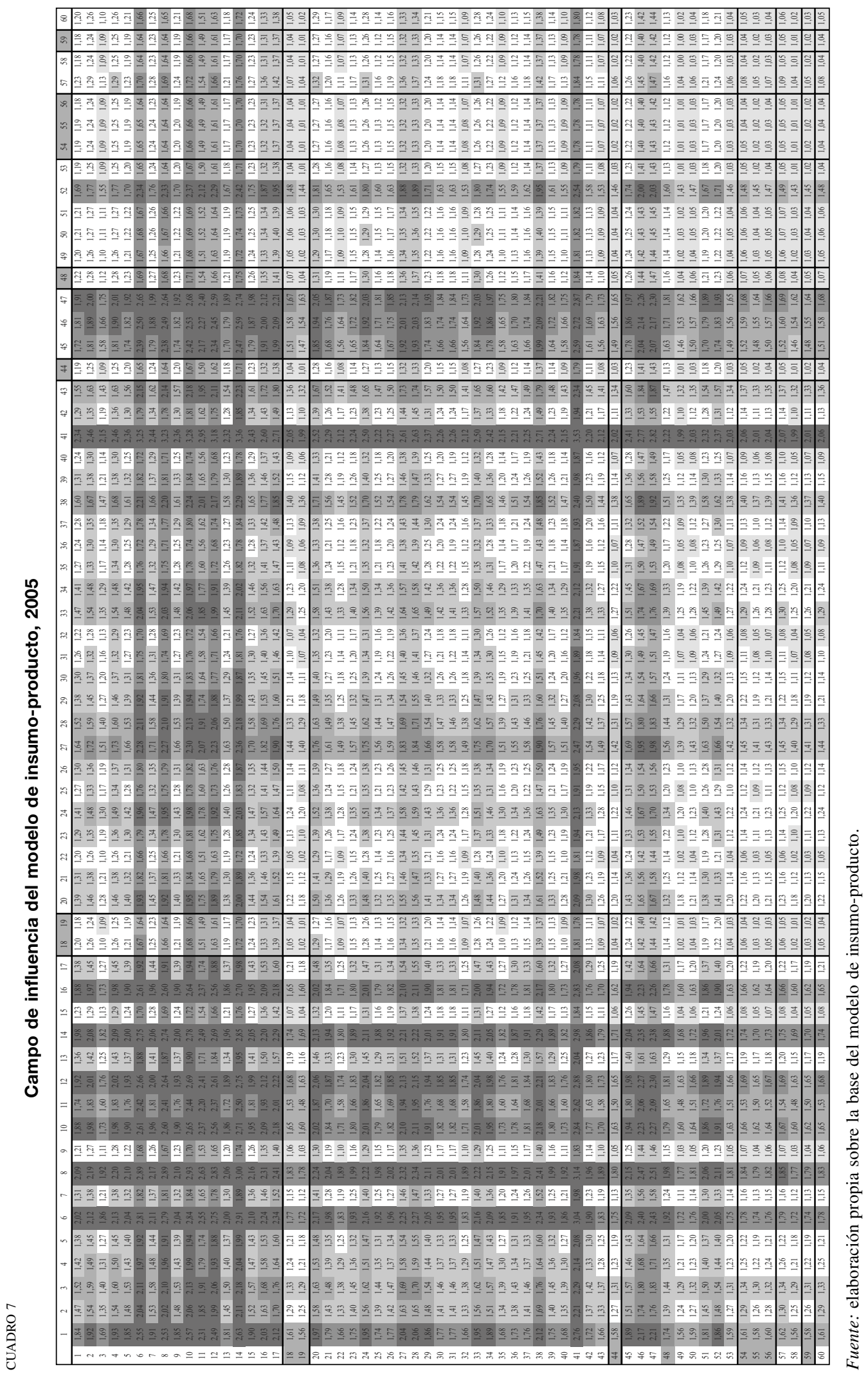




\section{VII \\ Consideraciones finales}

El presente trabajo contribuye al estudio del área de economía de la salud mediante la construcción de una matriz de insumo-producto con desagregación sectorial de los sectores analizados, que permite la evaluación de su eslabonamiento productivo. La compatibilización e integración de la matriz de insumo-producto con el Sistema de Cuentas Nacionales de Salud con respecto a los años 2000 y 2005 abre una importante agenda de investigación en el área de economía de la salud, pues permite el análisis y la evaluación de las políticas públicas en dicha área. Para comprender mejor las reformas del sistema de salud brasileño se deben tener en cuenta sus efectos macroeconómicos en las cadenas productivas y la generación de empleo, habida cuenta de la relevancia del sector desde el punto de vista del bienestar individual y social.

La amplia caracterización del sector de salud y su inserción en la estructura productiva realizada en este trabajo proporciona algunos resultados generales inherentes a la dinámica de ese sector en el Brasil. En primer lugar destaca la concentración de las relaciones intersectoriales en los sectores de salud, pues la mayor parte de las ventas se concentran en la demanda final de las familias o en el consumo intermedio del propio sector. Esto se traduce en escasos efectos de eslabonamientos regresivos y progresivos. Otras dos características de la estructura productiva del sector son el uso bastante intensivo del factor trabajo y su orientación a la generación de servicios médicos propiamente dichos. Los sectores de salud ligados a la producción de fármacos y aparatos médicos son una excepción a ese perfil y se asemejan más a los sectores industriales. Se trata, por lo tanto, de los sectores de salud con mayores eslabonamientos internos y externos.

Entre otras características estructurales del sector de salud en los años 2000 y 2005, se señala que los principales destinos de la producción del sector de fabricación de fármacos son el consumo intermedio y el consumo de las familias, y que dicho sector requiere una gran cantidad de insumos importados con respecto a los demás sectores de salud. Con respecto a los multiplicadores de empleo, hay sectores de salud con un alto efecto multiplicador, como en el caso de los servicios sociales privados (cuarto lugar en la clasificación), y sectores con un bajo efecto multiplicador, como por ejemplo, la fabricación de fármacos (posición número 49 en 2005).

Por último, otro resultado que se evidencia se refiere al eslabonamiento productivo de los sectores de salud. Mediante el campo de influencia se pudo apreciar que el sector de fabricación de productos farmacéuticos, que presenta el mayor grado de relación con otros sectores, tiene marcados eslabonamientos con los sectores de alimentos y bebidas, textil, celulosa y productos de papel, electricidad y gas, agua, alcantarillado y limpieza urbana. Este resultado explicita la cadena productiva de este sector de salud e ilustra su probable repercusión en la estructura productiva de la economía como consecuencia, entre otras cosas, de políticas de incentivo a la fabricación de productos farmacéuticos.

Desde el punto de vista de las políticas sectoriales a largo plazo, los resultados sugieren que los efectos de un aumento general de los gastos en salud en la estructura productiva del país son menos intensos que la media de los demás sectores de la economía, debido al bajo grado de eslabonamiento regresivo y progresivo del sector de salud. Esos efectos pueden ser más importantes si se concentran en los sectores de fármacos y atención hospitalaria. El envejecimiento de la población probablemente se traducirá en un incremento de los gastos, sobre todo en esos sectores. Este trabajo, basado en la compatibilización de la matriz de insumoproducto brasileña, representa un paso importante para permitir ese tipo de investigación.

De ese modo, el tratamiento del sector de salud en su carácter sistémico, es decir, evaluando su interdependencia con el tejido productivo de una economía como la brasileña, constituye una agenda de investigación nueva en un país que está sufriendo modificaciones — como la transición demográfica y las variaciones en la canasta de consumo de los individuos y la esperanza de vida - que pueden repercutir en el comportamiento de dicho sector. Este trabajo tiene un carácter seminal para la economía brasileña, pues constituye un punto de partida con miras a ampliar la línea de investigación y utilizar la matriz aquí desarrollada para calibrar modelos de equilibrio general computable y, por ejemplo, realizar un análisis del bienestar. 
ANEXO I

Procedimiento adoptado para la compatibilización de las matrices de insumo-producto del Brasil

El primer paso en la preparación de los datos consistió en la apertura de la matriz de recursos de bienes y servicios para incorporar los sectores de salud. Se obtuvo una matriz de 110x60. Con ese fin, se utilizó la matriz de recursos de bienes y servicios de las Cuentas Nacionales de Salud, suponiendo la misma tecnología de producción para todos los productos de una misma industria. De acuerdo con esa hipótesis, se construyó la matriz de actividad x producto (cuota de mercado o matriz D), cuyos coeficientes se obtienen por medio de la normalización de sus valores con respecto al total producido de cada producto. La matriz resultante brinda datos sobre la proporción de cada producto originado en los diversos sectores de actividad económica.

La segunda etapa fue la apertura de la matriz de oferta y demanda de la producción a precios básicos para incorporar el sector de salud. Para ello se utilizó el cuadro de usos de bienes y servicios de las Cuentas Satélite de dicho sector. Se obtuvo de ese modo una matriz de flujos monetarios con tecnología producto $\mathrm{x}$ sector (110x60), flujos de consumo intermedio y valores referentes a la demanda final.

El último paso consistió en transformar la matriz de oferta y demanda de la producción (construida por medio de la segunda etapa) en una matriz cuadrada. Para ello se adoptó el siguiente procedimiento: multiplicación de la matriz de cuota de mercado (construida sobre la base del primer paso) traspuesta, o sea, una matriz de 60x110, por la matriz de oferta y demanda de la producción (construida en b), es decir, una matriz de 110x60. Se obtuvo así una matriz cuadrada de 60×60. En la matriz de insumo-producto de 60x60 se incorporó el valor agregado, que se extrajo de la matriz de usos de bienes y servicios a precios al consumidor. Para los datos del valor agregado relativos a la salud se utilizó el cuadro de usos de bienes y servicios de las Cuentas Nacionales de Salud. Con respecto al cierre de la matriz desde el punto de vista de las compras, el valor de las importaciones se calculó como la diferencia entre el valor del total de la producción y la suma del consumo intermedio y el valor agregado.

Las estadísticas disponibles para el sector de salud en el Brasil a partir de las Cuentas Satélite son bastante detalladas. Hay datos relativos a la demanda (consumo final, consumo de la administración pública, inversión, gobierno) y al valor agregado, datos relativos al uso de insumos nacionales e importados, cuadros relativos a la producción de las actividades y desagregación por márgenes e impuestos.

Gracias a ello es posible desagregar la matriz y construir coeficientes técnicos teniendo en cuenta las especificidades de los productos y sectores. Los cuadros relativos al sector de salud en el Brasil, provenientes de las Cuentas Satélite, permiten captar la orientación de las ventas de productos y sectores, tanto para consumo intermedio como para consumo final. Esto es de suma importancia en el presente trabajo, pues la desagregación propuesta presenta algunos sectores más orientados al consumo intermedio y otros a la demanda final (por ejemplo, los servicios sociales privados).

Para tener una mejor idea sobre la estructura de las cuentas se presenta un extracto a continuación. 
Producción de las actividades (precios del año anterior en millones de reales)

Descripción del producto
Fabricación de aparatos para uso médicohospitalario y odontológico
Comercio de productos farmacéuticos, médicos, ortopédicos y odontológicos

\begin{tabular}{|c|c|c|c|c|c|}
\hline Productos farmoquímicos & 310 & 0 & 0 & 0 & 0 \\
\hline Medicamentos para uso humano & 12307 & 0 & 0 & 0 & 13 \\
\hline Medicamentos para uso veterinario & 1650 & 0 & 0 & 0 & 0 \\
\hline Materiales para uso médico, hospitalario y odontológico & 653 & 14 & 0 & 0 & 0 \\
\hline $\begin{array}{l}\text { Aparatos e instrumentos para uso médico, hospitalario y } \\
\text { odontológico }\end{array}$ & 3 & 2644 & 0 & 0 & 0 \\
\hline $\begin{array}{l}\text { Comercio de productos farmacéuticos, médicos, } \\
\text { ortopédicos y odontológicos }\end{array}$ & 0 & 0 & 7541 & 0 & 0 \\
\hline Planes de salud —incluido el seguro de salud & 0 & 0 & 0 & 7561 & 0 \\
\hline Salud pública & 0 & 0 & 0 & 0 & 29506 \\
\hline Servicios de atención hospitalaria & 0 & 0 & 0 & 0 & 1200 \\
\hline Otros servicios relacionados con la atención de la salud & 0 & 0 & 0 & 0 & 8 \\
\hline Servicios sociales privados & 0 & 0 & 0 & 0 & 0 \\
\hline Agropecuaria & 0 & 0 & 0 & 0 & 0 \\
\hline Industria extractiva mineral & 0 & 0 & 0 & 0 & 0 \\
\hline Industria de transformación & 828 & 9 & 0 & 0 & 1 \\
\hline Producción y distribución de electricidad, gas y agua & 0 & 0 & 0 & 0 & 0 \\
\hline Construcción & 0 & 0 & 3 & 0 & 0 \\
\hline Comercio & 0 & 0 & 0 & 0 & 0 \\
\hline Transporte, almacenamiento y correo & 0 & 0 & 0 & 0 & 0 \\
\hline Servicios de información & 0 & 0 & 0 & 0 & 0 \\
\hline $\begin{array}{l}\text { Intermediación financiera, seguros y previsión } \\
\text { complementaria }\end{array}$ & 0 & 0 & 0 & 0 & 0 \\
\hline Actividades inmobiliarias y alquiler & 15 & 0 & 16 & 5 & 0 \\
\hline Otros servicios & 0 & 0 & 0 & 0 & 0 \\
\hline Administración, salud y educación públicas & 0 & 0 & 0 & 0 & 0 \\
\hline Ajuste $\mathrm{CIF} / \mathrm{FOB}$ & - & - & - & - & - \\
\hline Total & 15766 & 2667 & 7560 & 7566 & 30728 \\
\hline
\end{tabular}


Cuadro A.1 (conclusión)

Producción de las actividades (precios del año anterior en millones de reales)

Descripción del producto

$\begin{array}{lcccc}\text { Actividades } & \begin{array}{c}\text { Otras } \\ \text { actividades } \\ \text { de atención } \\ \text { hospitalaria }\end{array} & \begin{array}{c}\text { Servicios } \\ \text { con la atención } \\ \text { de la salud }\end{array} & \begin{array}{c}\text { Otras } \\ \text { privados }\end{array} & \begin{array}{c}\text { Total del } \\ \text { producto }\end{array} \\ \end{array}$

Productos farmoquímicos

Medicamentos para uso humano

Medicamentos para uso veterinario

Materiales para uso médico, hospitalario y odontológico

Aparatos e instrumentos para uso médico, hospitalario y

odontológico

Comercio de productos farmacéuticos, médicos,

ortopédicos y odontológicos

Planes de salud —incluido el seguro de salud

Salud pública

Servicios de atención hospitalaria

Otros servicios relacionados con la atención de la salud

Servicios sociales privados

Agropecuaria

Industria extractiva mineral

Industria de transformación

Producción y distribución de electricidad, gas y agua

Construcción

\section{Comercio}

Transporte, almacenamiento y correo

Servicios de información

Intermediación financiera, seguros y previsión

complementaria

Actividades inmobiliarias y alquiler

Otros servicios

Administración, salud y educación públicas

Ajuste CIF/FOB

0

0
0
0
0
0

245

$\begin{array}{rr}2 & 312 \\ 95 & 12415 \\ 245 & 1895 \\ 6 & 673 \\ 8 & 2655\end{array}$

Total

14290

23165

$0 \quad 0$

0

7541

Fuente: Instituto Brasileño de Geografía y Estadística (IBGE), Contas Satélites de Saúde.

CIF (por sus siglas en inglés): costo, seguro y flete.

FOB: incluye el valor de la mercancía y gastos de transporte al país de destino.

La estructura de desagregación de las Cuentas Satélite permite establecer las diferencias en la estructura de producción. Para verificar las diferencias tecnológicas se presenta un extracto de la matriz inversa de Leontief en que se ponen de relieve los sectores de salud. Es posible apreciar las particularidades "tecnológicas" de cada sector. 


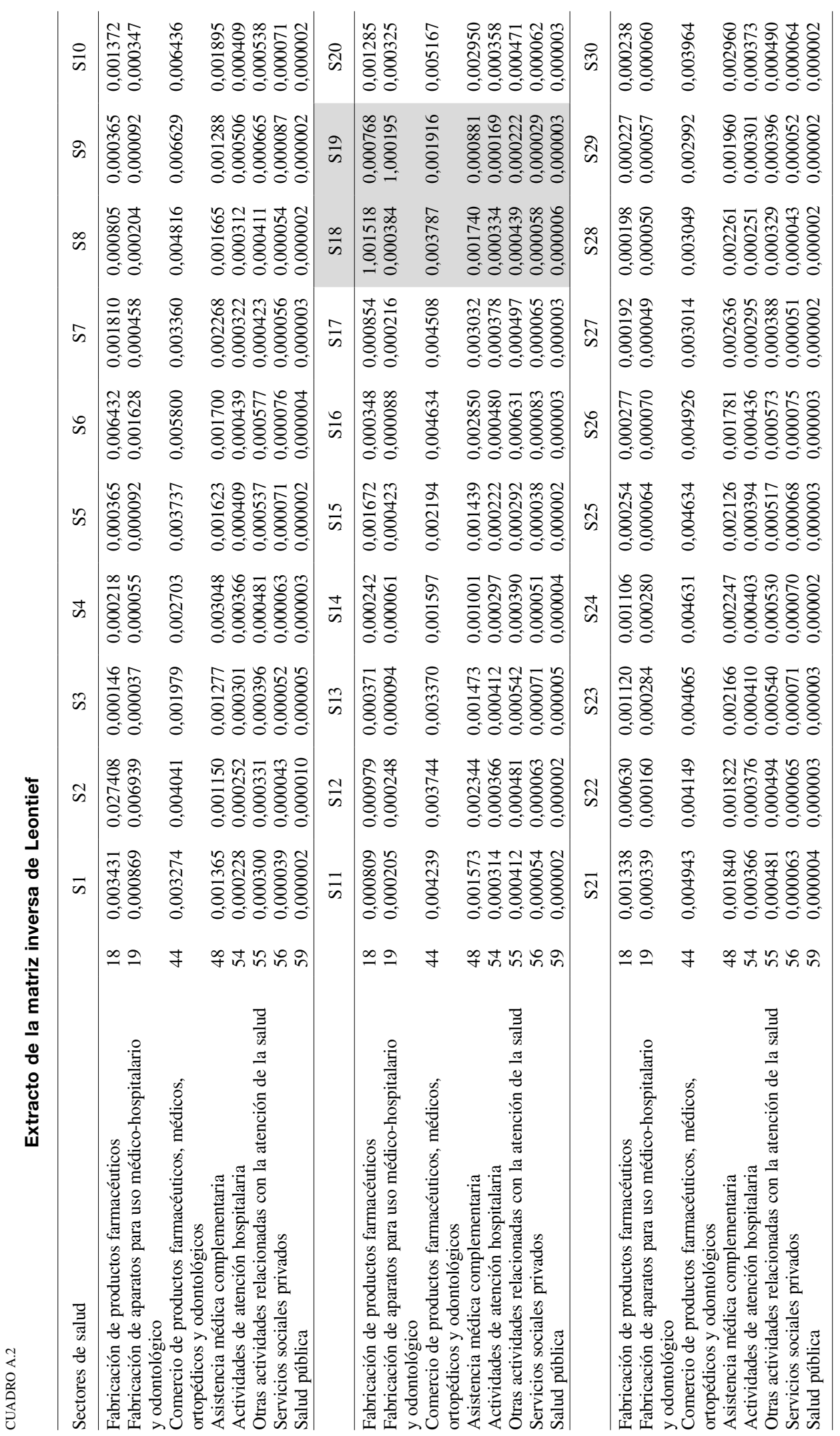




\begin{tabular}{|c|c|c|c|c|c|c|c|c|c|c|c|}
\hline 孚 & $\begin{array}{l}1 \\
0 \\
0 \\
0 \\
0 \\
0 \\
0\end{array}$ & 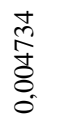 & 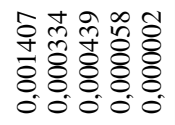 & $\ddot{n}$ & 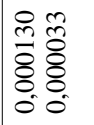 & $\begin{array}{l}\text { aे } \\
\text { ָे } \\
\text { ठ } \\
0\end{array}$ & 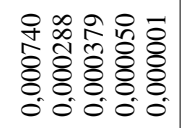 & $\begin{array}{l}\text { D } \\
\text { n }\end{array}$ & 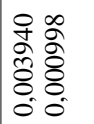 & 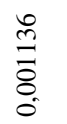 & 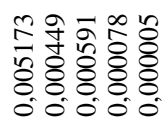 \\
\hline हे & $\begin{array}{l}\infty \\
0 \\
\hat{1} \\
0 \\
0 \\
0 \\
0 \\
0 \\
0 \\
0\end{array}$ & 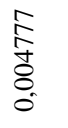 & 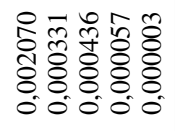 & 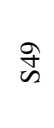 & 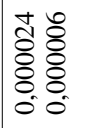 & 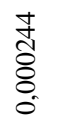 & 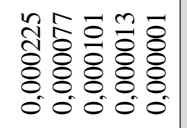 & $\hat{\tilde{n}}$ & 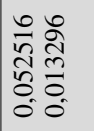 & 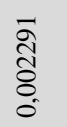 & 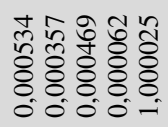 \\
\hline$\stackrel{\infty}{n}$ & 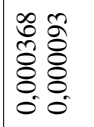 & $\begin{array}{l}2 \\
\hat{\alpha} \\
\vdots \\
0 \\
0\end{array}$ & 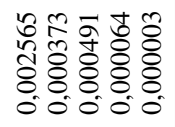 & 立 & 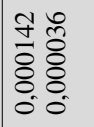 & 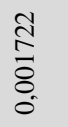 & 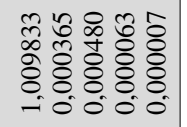 & $\begin{array}{l}\infty \\
\hat{n}\end{array}$ & 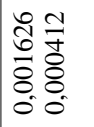 & 兽 & 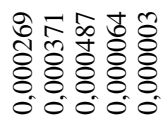 \\
\hline$\hat{\tilde{n}}$ & 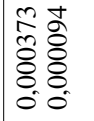 & 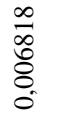 & 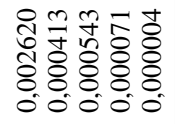 & 点 & 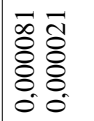 & $\begin{array}{l}0 \\
\& \\
8 \\
0 \\
0\end{array}$ & $\begin{array}{l}0 \\
0 \\
0 \\
0\end{array}$ & $\tilde{n}$ & 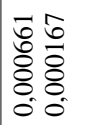 & $\frac{8}{10}$ & 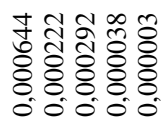 \\
\hline D & 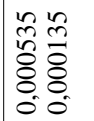 & $\begin{array}{l}\hat{N} \\
\hat{\tilde{o}} \\
0 \\
0\end{array}$ & 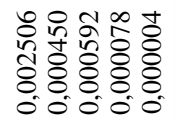 & 吕 & 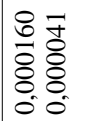 & $\frac{0}{0}$ & 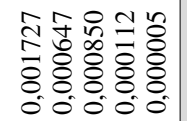 & ڤn & 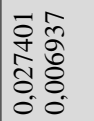 & 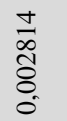 & 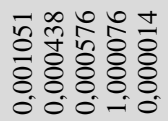 \\
\hline$\tilde{n}$ & 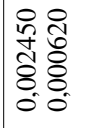 & 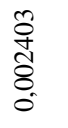 & 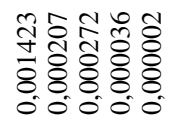 & if & 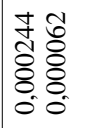 & $\begin{array}{l}\text { ते } \\
\text { ôे } \\
0 \\
0\end{array}$ & 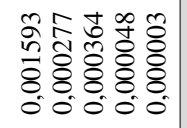 & $\tilde{n}$ & 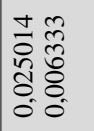 & $\begin{array}{l}\text { oे } \\
\text { ते } \\
\text { ठ. } \\
0\end{array}$ & 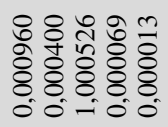 \\
\hline$\underset{m}{ \pm}$ & $\begin{array}{l}\text { în } \\
\text { స̃ } \\
0 \\
0 \\
0 \\
0 \\
0 \\
0\end{array}$ & 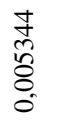 & 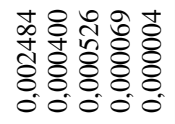 & 寺 & 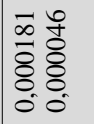 & 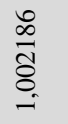 & 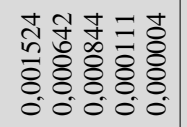 & $\begin{array}{l}\tilde{n} \\
\tilde{n}\end{array}$ & 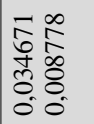 & 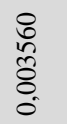 & 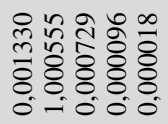 \\
\hline$\tilde{\tilde{n}}$ & 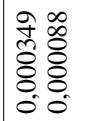 & $\begin{array}{l}\text { oे } \\
\text { o. } \\
8 \\
0 .\end{array}$ & 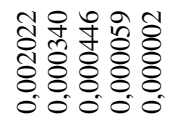 & 管 & 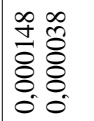 & $\begin{array}{l}\stackrel{0}{\infty} \\
\stackrel{0}{1} \\
0 \\
0\end{array}$ & 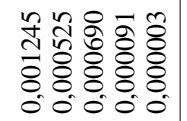 & $\tilde{n}$ & 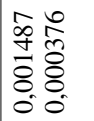 & $\begin{array}{l}\text { 年 } \\
\frac{1}{2} \\
8 \\
0 \\
0\end{array}$ & 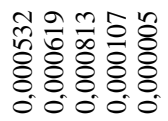 \\
\hline$\tilde{\tilde{n}}$ & 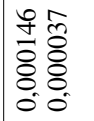 & 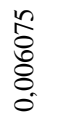 & 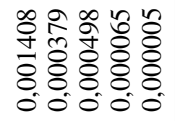 & F & 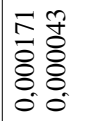 & $\begin{array}{l}m \\
\stackrel{9}{9} \\
\stackrel{+}{8} \\
0 \\
0\end{array}$ & 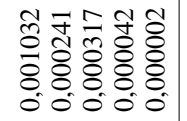 & $\begin{array}{l}\tilde{v} \\
\tilde{n}\end{array}$ & 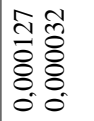 & $\begin{array}{l}\stackrel{\infty}{\infty} \\
\stackrel{\infty}{8} \\
0\end{array}$ & 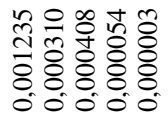 \\
\hline $\bar{n}$ & 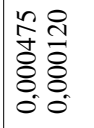 & $\begin{array}{l}\text { âे } \\
\vdots \\
0 \\
0 \\
0\end{array}$ & 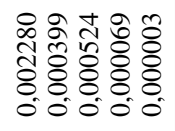 & $\vec{F}$ & $\begin{array}{l}\hat{a} \\
\overline{0} \\
0 \\
0 \\
0 \\
0 \\
0\end{array}$ & $\begin{array}{l}\hat{\tilde{\pi}} \\
\stackrel{0}{8} \\
0 \\
0\end{array}$ & 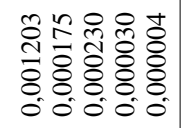 & $\bar{n}$ & 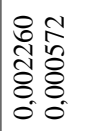 & $\begin{array}{l}\text { त. } \\
0 \\
0 \\
0 \\
0\end{array}$ & 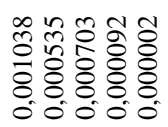 \\
\hline & 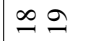 & 寸 & oin & & 9 & 寸 & ñ in in & & $\stackrel{\infty}{=}$ & 寸 & in in \\
\hline 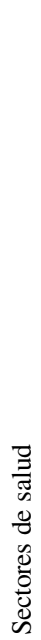 & 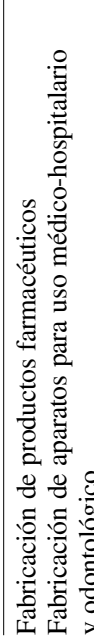 & 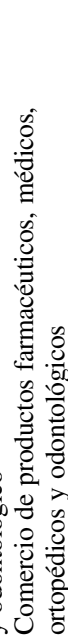 & 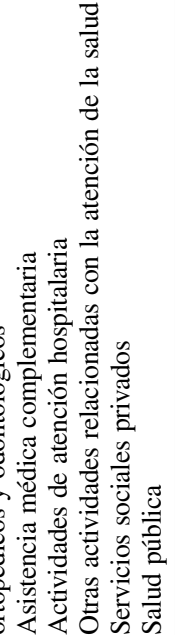 & & 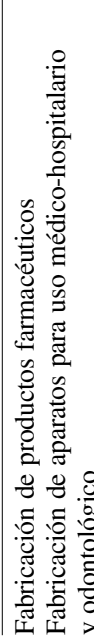 & 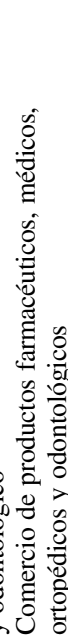 & 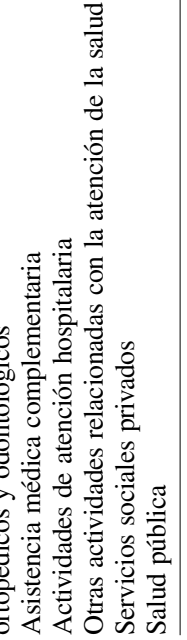 & & 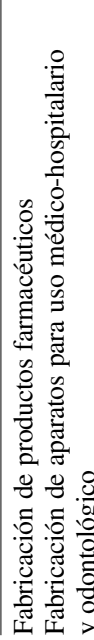 & 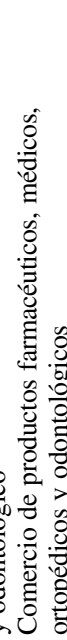 & 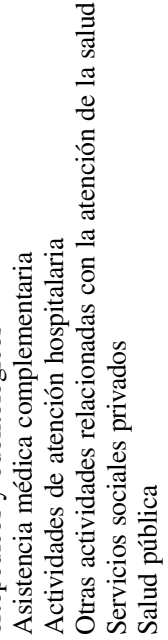 \\
\hline
\end{tabular}


ANEXO II

\section{Formalización de los indicadores de insumo-producto}

Los multiplicadores complementan el análisis de la importancia de determinado sector en la economía, pues permiten evaluar los efectos que se producen a corto y largo plazo en un determinado sistema económico a raíz de perturbaciones exógenas (Miller y Blair, 2009).

El multiplicador de producción corresponde a una variación directa e indirecta de la producción total de la economía de todos los sectores y regiones, debida a la variación exógena de una unidad monetaria de la demanda final de una región de un determinado sector. En términos formales, el multiplicador de producto simple para el sector $j, O_{j}$, está dado por:

$$
O_{j}=\sum_{i=1}^{n} b_{i j}
$$

donde $b_{i j}$ son los elementos de la matriz inversa de Leontief.

El multiplicador del empleo estima los efectos de una variación exógena en la demanda final, o sea, el número de empleos que se generan en la economía, directa e indirectamente, debido a una variación en la demanda final suficiente para causar el aumento de un empleo en el sector $j$. Para calcular el multiplicador de empleo se debe estimar la relación entre el valor de la producción de un determinado sector y el empleo en ese sector. En términos formales:

$$
w_{n+1, j}=e_{j} / X_{j}
$$

donde $e_{j}$ corresponde al personal ocupado en el sector $j$ y $X_{j}$ es el valor bruto de la producción del sector $j$.

Para una economía con $n$ sectores:

$$
W_{R}=\left\lfloor w_{n+1,1}, w_{n+1,2}, \cdots, w_{n+1, n}\right\rfloor
$$

Por lo tanto, el multiplicador simple de empleo estará dado por:

$$
E_{j}=\sum_{i=1}^{n} w_{n+1, i} b_{i j}
$$

en que $i$ representa un determinado sector de la economía y $w_{n+1, i}$ es la razón entre el número de ocupados y el valor de producción.

La matriz resultante de esa transformación $\left(E_{j}\right)$ proporciona la capacidad sectorial de generación de empleo por unidad adicional de demanda final. Cabe resaltar que la estructura de la matriz $E_{j}$ es semejante a la estructura de las matrices B (Leontief) y A (matriz de los coeficientes). Por consiguiente, para cada sector $j$, la suma de los elementos de cada columna representa el multiplicador de empleo del sector $j$.

Rasmussen (1952) y Hirschman (1958) utilizan los índices de eslabonamiento regresivo y progresivo para establecer los sectores que tendrían el mayor poder de eslabonamiento en la economía. Los eslabonamientos regresivos (poder de dispersión) $-U_{j}$ - determinan cuánto demanda un sector de los demás sectores de la economía, mientras que los eslabonamientos progresivos (sensibilidad de la dispersión) $-U_{i}-$ determinan la medida en que ese sector es demandado por los demás sectores de la economía. El índice de eslabonamiento regresivo se define como:

$U_{j}=\frac{1}{n} \sum_{i} b_{i j} / \frac{1}{n^{2}} \sum_{i} \sum_{j} b_{i j} \quad(i, j=1,2, \ldots, n)(\mathrm{A} .5)$

El índice de eslabonamiento progresivo se define como:

$U_{i}=\frac{1}{n} \sum_{j} b_{i j} / \frac{1}{n^{2}} \sum_{i} \sum_{j} b_{i j} \quad(i, j=1,2, \ldots, n)($ A.6 $)$

Si $U_{j}>1$ indica que una variación unitaria en la demanda final del sector $j$ crea un aumento por sobre la media en la economía. Si $U_{i}>1$ indica que una variación unitaria en la demanda final de todos los sectores crea un aumento por sobre la media en el sector. Los valores superiores a 1 en ambos índices indican sectores clave en la economía. Esos sectores poseen fuertes efectos de eslabonamiento en términos del flujo de bienes y servicios y sus aportes al crecimiento de la economía están por sobre la media. 
Brasil: índice de eslabonamientos y sectores clave, 2000 y 2005

\begin{tabular}{|c|c|c|c|c|c|c|}
\hline \multirow{2}{*}{ Sectores económicos } & \multicolumn{2}{|c|}{$\begin{array}{l}\text { Eslabonamientos } \\
\text { regresivos }\end{array}$} & \multicolumn{2}{|c|}{$\begin{array}{l}\text { Eslabonamientos } \\
\text { progresivos }\end{array}$} & \multicolumn{2}{|c|}{ Sector clave } \\
\hline & 2000 & 2005 & 2000 & 2005 & 2000 & 2005 \\
\hline Agricultura, silvicultura, explotación forestal & 0,8673 & 0,9086 & 1,6158 & 1,7715 & - & - \\
\hline Ganadería y pesca & 0,9629 & 1,0238 & 0,8550 & 0,8492 & - & - \\
\hline Petróleo y gas natural & 0,9220 & 0,9692 & 1,3107 & 1,5526 & - & - \\
\hline Mineral de hierro & 1,0807 & 1,0224 & 0,6876 & 0,7293 & - & - \\
\hline Alimentos y bebidas & 1,2718 & 1,2796 & 1,3742 & 1,4364 & $\mathrm{X}$ & $\mathrm{X}$ \\
\hline Productos del tabaco & 1,1258 & 1,2408 & 0,5625 & 0,5469 & - & - \\
\hline Textiles & 1,0525 & 1,0254 & 1,1146 & 1,0826 & $\mathrm{X}$ & $\mathrm{X}$ \\
\hline Prendas de vestir y accesorios & 1,0082 & 1,0241 & 0,5989 & 0,5657 & - & - \\
\hline Artículos de cuero y calzado & 1,2511 & 1,2159 & 0,6824 & 0,6822 & - & - \\
\hline Productos de madera - excepto muebles & 1,0114 & 1,1063 & 0,8078 & 0,8406 & - & - \\
\hline Celulosa y productos de papel & 1,0942 & 1,1403 & 1,2073 & 1,1404 & $X$ & $\mathrm{X}$ \\
\hline Diarios, revistas y discos & 0,9844 & 0,9532 & 1,0359 & 0,8628 & - & - \\
\hline Productos químicos & 1,1452 & 1,0975 & 1,9503 & 2,1663 & $\mathrm{X}$ & $\mathrm{X}$ \\
\hline Fabricación de resina y elastómeros & 1,3315 & 1,2331 & 1,0811 & 1,1639 & $\mathrm{X}$ & $\mathrm{X}$ \\
\hline Fabricación de productos farmacéuticos & 0,9313 & 0,9292 & 0,7023 & 0,6315 & - & - \\
\hline Fabricación de aparatos para uso médico-hospitalario y odontológico & 0,7266 & 0,7272 & 0,5637 & 0,5485 & - & - \\
\hline Pesticidas & 1,2635 & 1,1579 & 0,7379 & 0,7721 & - & - \\
\hline Perfumería, higiene y limpieza & 1,0712 & 1,1283 & 0,7093 & 0,6286 & - & - \\
\hline Pinturas, barnices, esmaltes y lacas & 1,1129 & 1,0757 & 0,6563 & 0,6185 & - & - \\
\hline Productos y preparados químicos diversos & 1,0946 & 1,0787 & 0,8872 & 0,8494 & - & - \\
\hline Artículos de caucho y plástico & 1,2183 & 1,1675 & 1,2801 & 1,3594 & $\mathrm{X}$ & $\mathrm{X}$ \\
\hline Cemento & 1,0467 & 1,1025 & 0,6274 & 0,6087 & - & - \\
\hline Otros productos de minerales no metálicos & 1,1245 & 1,0734 & 0,7881 & 0,7804 & - & - \\
\hline Fabricación de acero y derivados & 1,0944 & 1,0989 & 1,3294 & 1,6753 & $\mathrm{X}$ & $\mathrm{X}$ \\
\hline Metalurgia de metales no ferrosos & 1,0882 & 1,0197 & 0,9302 & 0,8489 & - & - \\
\hline Productos de metal - excepto máquinas y equipos & 1,0836 & 1,0515 & 1,1956 & 1,3435 & $\mathrm{X}$ & $\mathrm{X}$ \\
\hline Máquinas y equipos, incluidos mantenimiento y reparaciones & 1,1059 & 1,1290 & 0,9712 & 0,9253 & - & - \\
\hline Electrodomésticos & 1,2088 & 1,2245 & 0,5708 & 0,5478 & - & - \\
\hline Máquinas para oficina y equipos de informática & 0,8997 & 0,9179 & 0,5525 & 0,5306 & - & - \\
\hline Aparatos e instrumentos médico-hospitalarios, de medición y ópticos & 0,8435 & 0,8426 & 0,6242 & 0,6008 & - & - \\
\hline Automóviles, camionetas y vehículos utilitarios & 1,1507 & 1,2981 & 0,5815 & 0,5500 & - & - \\
\hline Camiones y omnibuses & 1,0829 & 1,1955 & 0,5731 & 0,5733 & - & - \\
\hline Piezas y accesorios para vehículos automotores & 1,1286 & 1,2395 & 0,9680 & 1,2576 & - & - \\
\hline Otros equipos de transporte & 0,8862 & 1,1223 & 0,5860 & 0,6755 & - & - \\
\hline Muebles y productos de industrias diversas & 1,0493 & 1,0252 & 0,6573 & 0,6066 & - & - \\
\hline Electricidad y gas, agua, alcantarillado y limpieza urbana & 0,9196 & 0,8993 & 2,2950 & 2,5283 & - & - \\
\hline Construcción & 0,9552 & 0,8919 & 0,7809 & 0,7343 & - & - \\
\hline Comercio de otros servicios de salud & 0,7532 & 0,7443 & 2,4336 & 2,5043 & - & - \\
\hline $\begin{array}{l}\text { Comercio de productos farmacéuticos, médicos, ortopédicos y } \\
\text { odontológicos }\end{array}$ & 0,8068 & 0,7945 & 0,6318 & 0,6322 & - & - \\
\hline Transporte, almacenamiento y correo & 0,9298 & 0,9719 & 2,5105 & 2,5416 & - & - \\
\hline Servicios de información & 0,9017 & 0,8753 & 1,9773 & 1,9822 & - & - \\
\hline Intermediación financiera y otros seguros & 0,8835 & 0,7574 & 2,2145 & 1,8974 & - & - \\
\hline Asistencia médica complementaria & 0,9767 & 0,9343 & 0,6406 & 0,5811 & - & - \\
\hline Servicios inmobiliarios y alquiler & 0,5732 & 0,5661 & 0,8905 & 0,8335 & - & - \\
\hline Servicios de mantenimiento y reparación & 0,7837 & 0,7400 & 0,7956 & 0,6801 & - & - \\
\hline Servicios de alojamiento y alimentación & 1,0738 & 1,0632 & 0,7704 & 0,7014 & - & - \\
\hline Servicios prestados a las empresas & 0,8680 & 0,8112 & 2,4883 & 2,2097 & - & - \\
\hline Educación mercantil & 0,8144 & 0,8221 & 0,5717 & 0,5511 & - & - \\
\hline Actividades de atención hospitalaria & 0,9960 & 0,9828 & 0,5443 & 0,5317 & - & - \\
\hline Otras actividades relacionadas con la atención de salud & 0,8197 & 0,8540 & 0,5513 & 0,5353 & - & - \\
\hline Servicios sociales privados & 0,9467 & 0,8858 & 0,5356 & 0,5223 & - & - \\
\hline Otros servicios & 0,8480 & 0,8188 & 0,8438 & 0,7459 & - & - \\
\hline Educación pública & 0,6919 & 0,6963 & 0,5375 & 0,5233 & - & - \\
\hline Salud pública & 0,8347 & 0,8441 & 0,5334 & 0,5205 & - & - \\
\hline Administración pública y seguridad social & 0,8268 & 0,8008 & 0,7167 & 0,6672 & - & - \\
\hline
\end{tabular}

Fuente: elaboración propia.

Nota: sectores de salud en sombreado. 


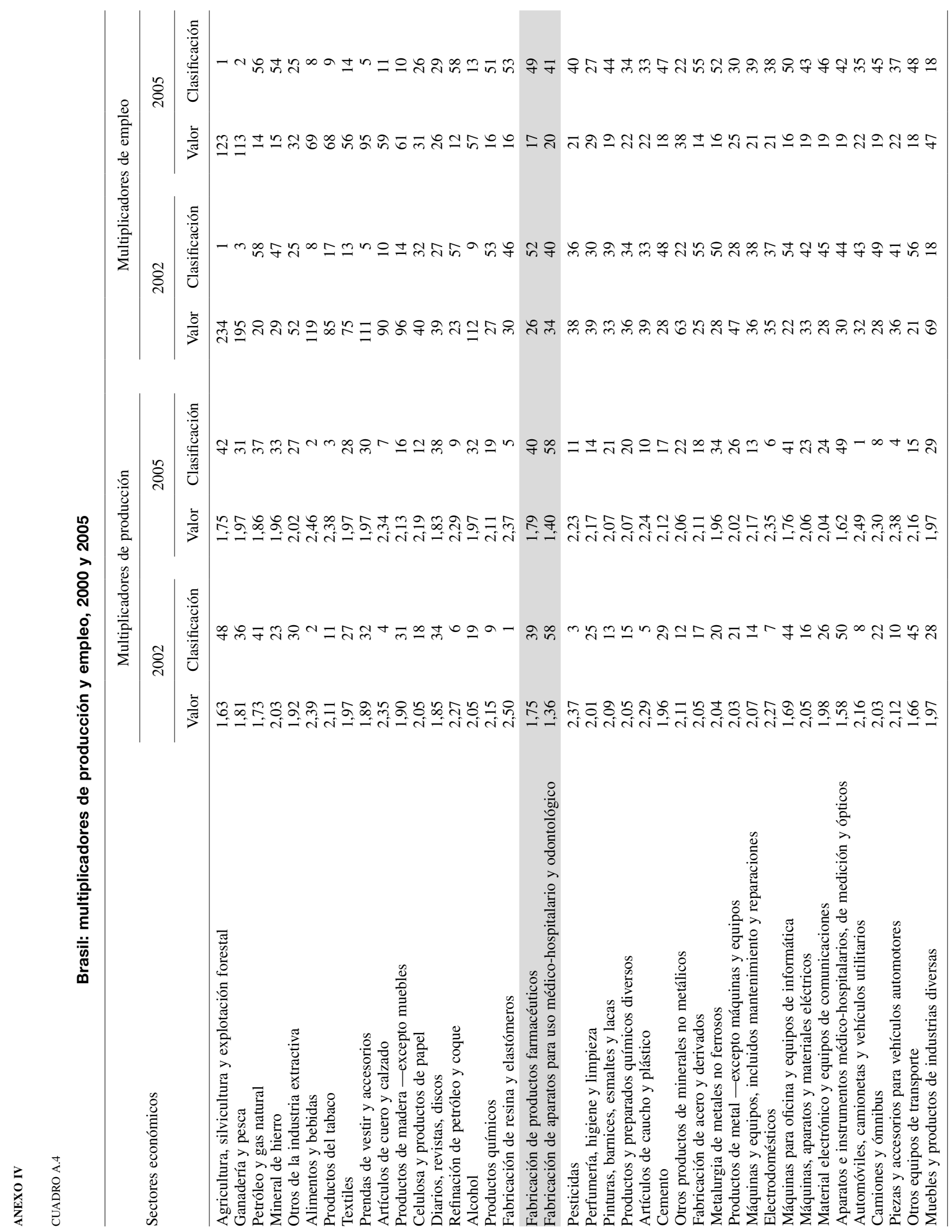




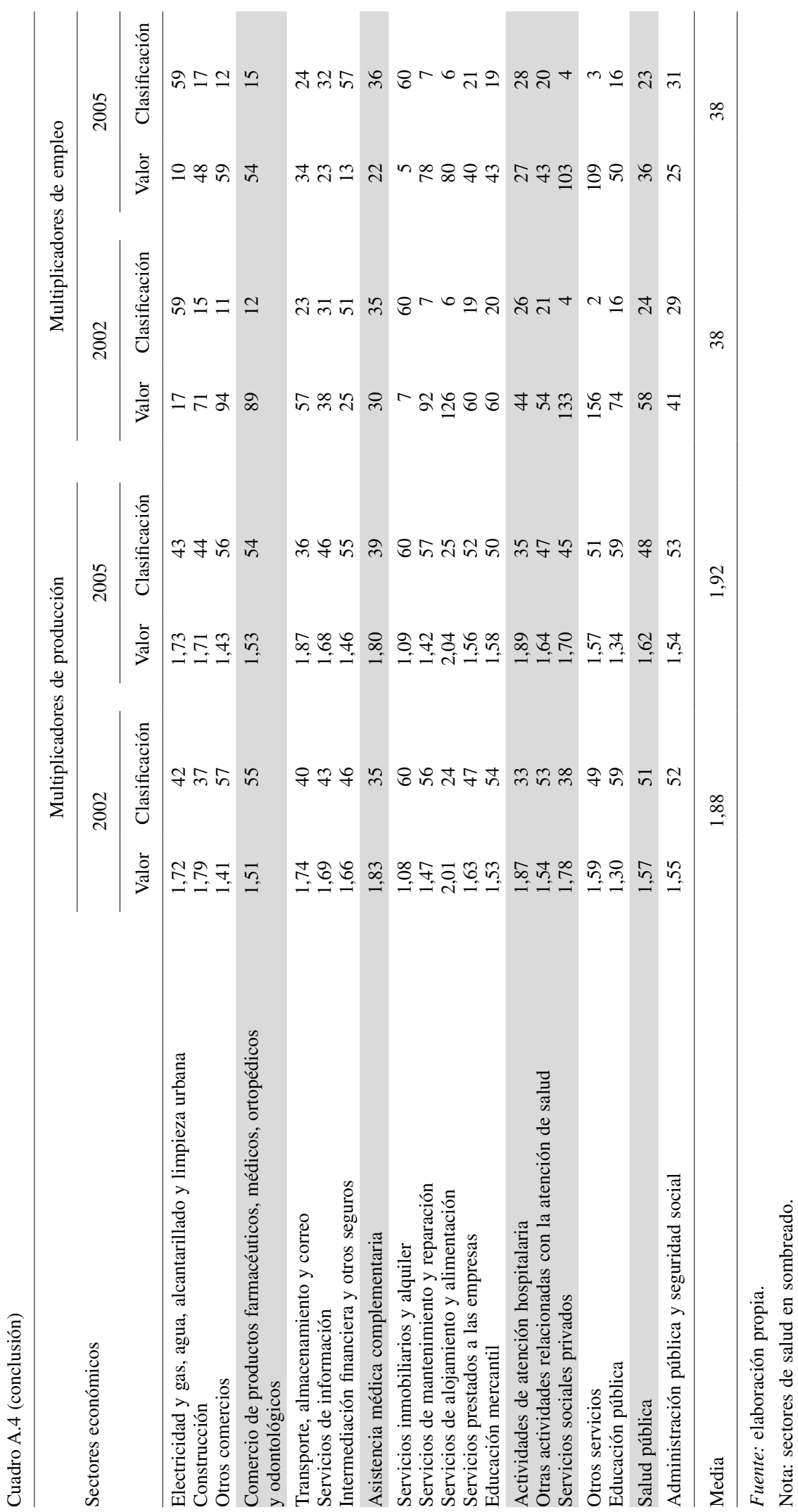




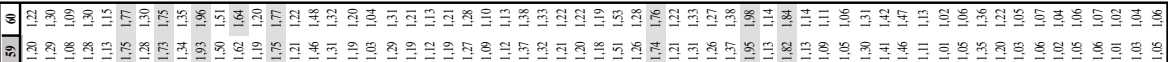

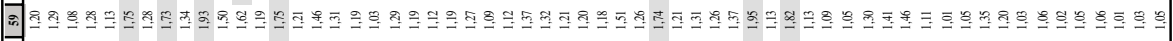

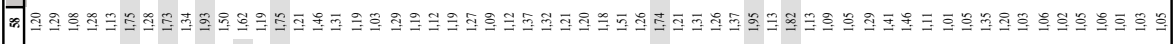

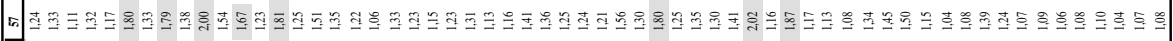

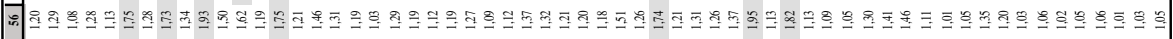

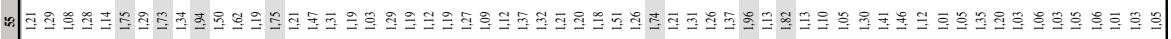

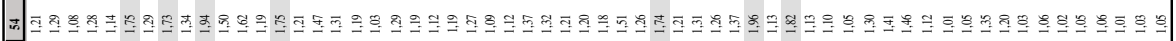

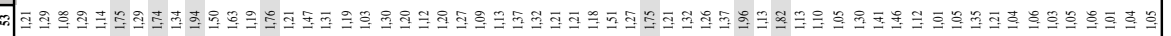

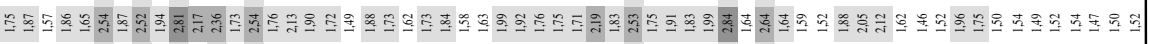

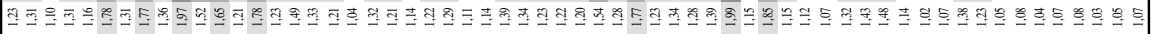

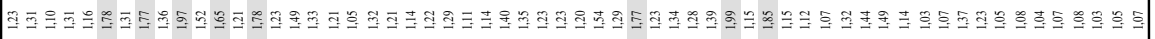

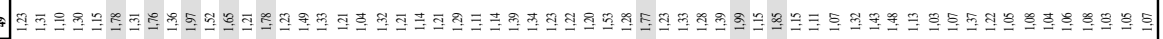

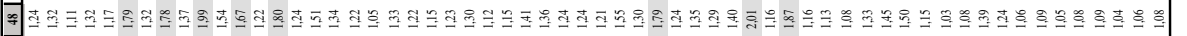

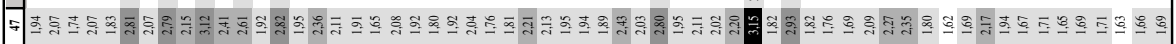
F

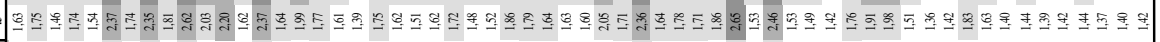

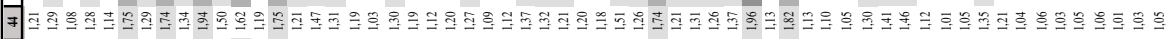

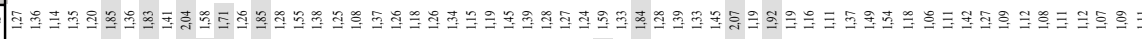

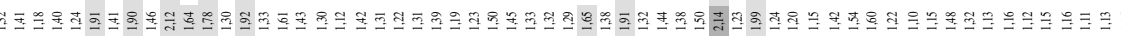

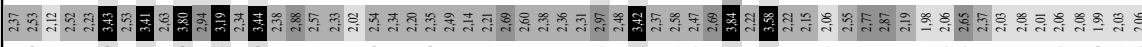

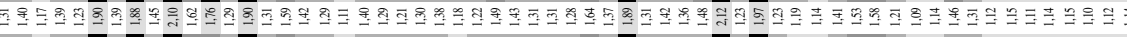

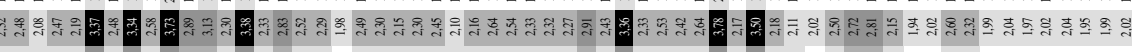

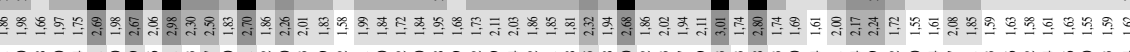

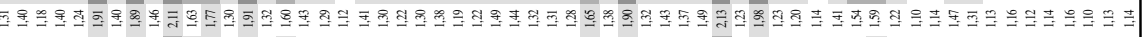

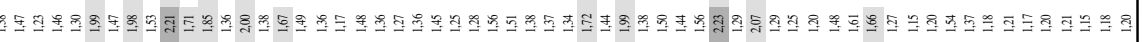

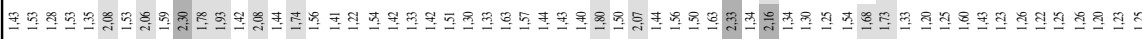

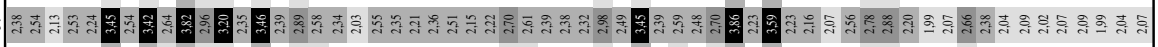

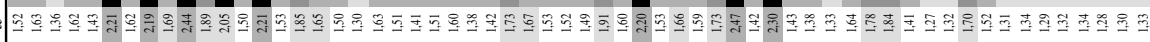

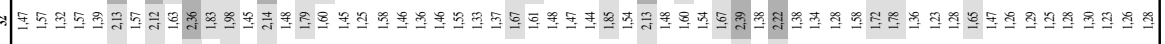

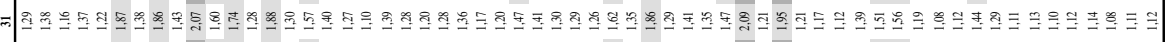

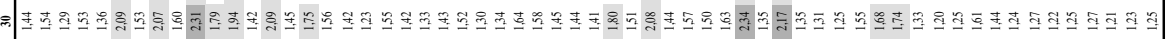

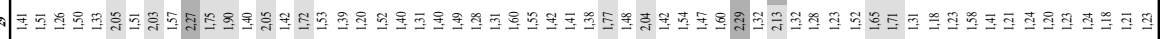

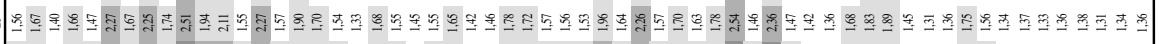

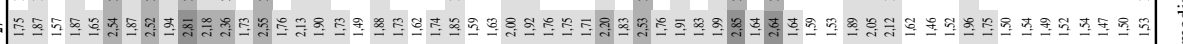

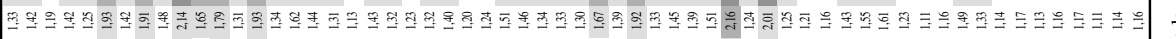

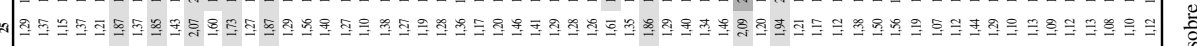

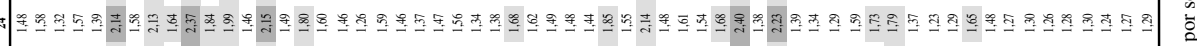

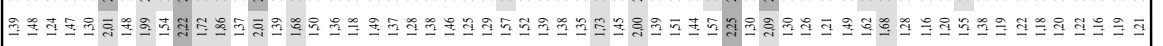

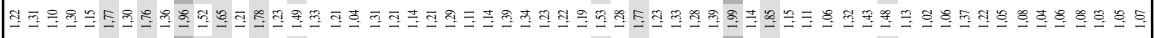

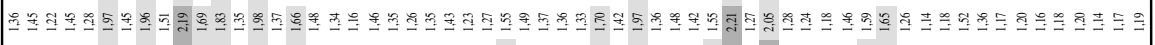

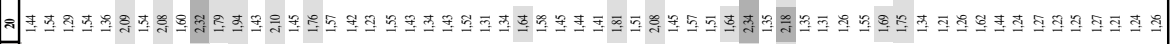

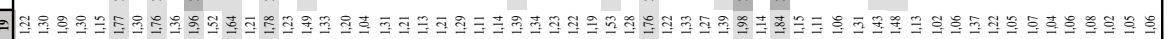

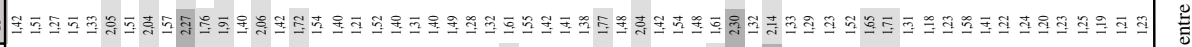

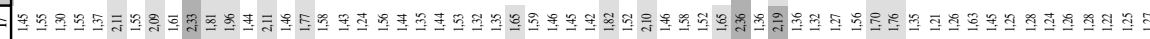

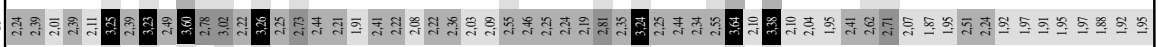

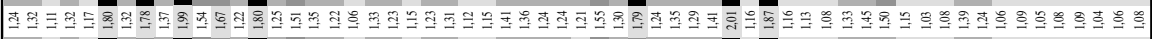

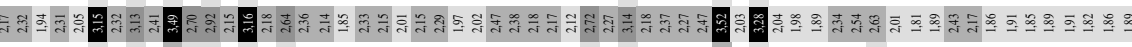

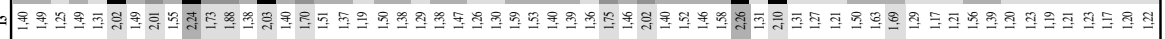

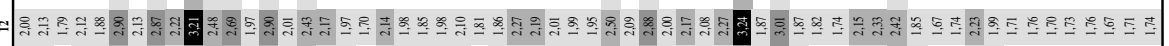

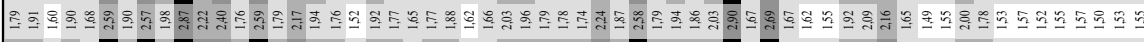

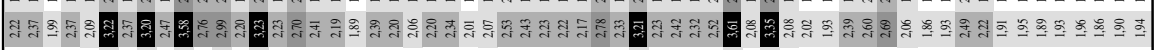

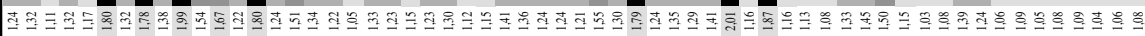

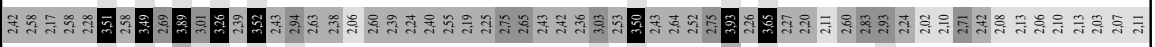

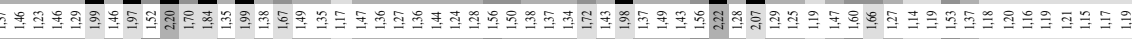

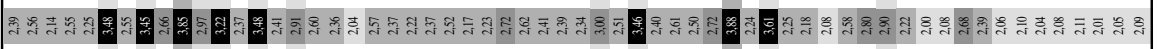

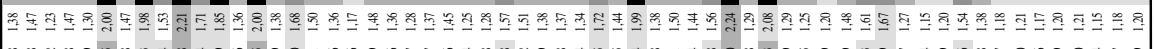

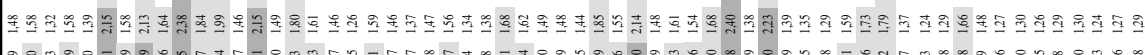

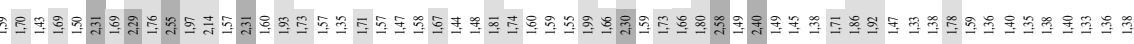

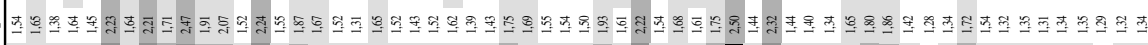

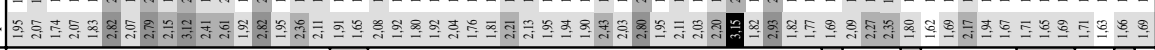

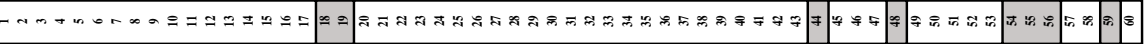

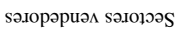




\section{Bibliografía}

Almeida, C. y otros (2000), "Health sector reform in Brazil: A case study of inequity", International Journal of Health Services, vol. $30, \mathrm{~N}^{\circ} 1$, SAGE.

Andrade, M.V. (2002), "A saúde na PNAD", Texto para Discussão, $\mathrm{N}^{\circ} 170$, Belo Horizonte, Universidad Federal de Minas Gerais/ Centro de Desarrollo y Planificación Regional (CEDEPLAR).

Azzoni, C.R. y otros (2007), "Social policies, personal and income inequality in Brazil: I-O analysis of the "Bolsa Família" progam", Anais do V Encontro Nacional da Associação Brasileira de Estudos Regionais e Urbanos, Pernambuco.

Banco Mundial (2007), "PIB per cápita" [en línea] http://datos. bancomundial.org/indicador/NY.GDP.PCAP.CD.

Betarelli Junior, A.A., S.Q.A. Bastos y F.S. Perobelli (2008), "As pressões das exportações setoriais sobre os modais de transporte: uma abordagem híbrida e intersetorial de insumoproduto", Pesquisa e Planejamento Econômico, vol. 38, № 3 , Instituto de Investigación Económica Aplicada (IPEA).

Bloom, D., D. Canning y J. Sevilla (2001), "The effect of health on economic growth: Theory and evidence", NBER Working Paper, $\mathrm{N}^{\circ}$ 8587, Cambridge, Massachusetts, National Bureau of Economic Research.

Brasil, Ministerio de Salud (2010), Doenças infecciosas e parasitárias, Brasilia.

(2004), Saúde Brasil 2004: uma análise da situação de saúde, Brasilia.

Camargo, M.C.S., R.N. Rodrigues y C.J. Machado (2006), "Expectativa de vida saudável para idosos brasileiros, 1998 e 2003", Anais do XV Encontro Nacional de Estudos Populacionais, Caxambu, Minas Gerais.

Campelo, V., M.A.G. Gonçalves y E.A. Donadi (2005), "Mortalidade por doenças infecciosas e parasitárias no Município de Teresina-PI (Brasil), 1971-2000”, Revista Brasileira de Epidemiologia, vol. 8, $\mathrm{N}^{\circ}$ 1, Asociación Brasileña de Salud Colectiva.

Correa, H. y B. Parker (2005), "An application of organizational input-output analysis to hospital management", SocioEconomic Planning Sciences, vol. 39, N 4, Amsterdam, Elsevier.

Guilhoto, J.J.M. (2004), Análise de insumo-produto: teoria e fundamentos, São Paulo.

Guilhoto, J.J.M. y otros (1994), "Índices de ligação e setores-chave na economia brasileira: 1959/80", Pesquisa e Planejamento Econômico, vol. 24, $\mathrm{N}^{\circ}$ 4, Instituto de Investigación Económica Aplicada (IPEA).

Hilgemberg, E.M. y J.M. Guilhoto (2006), "Uso de combustíveis e emissões de $\mathrm{CO} 2$ no Brasil: um modelo inter-regional de insumo-produto", Nova Economia, vol. 16, $\mathrm{N}^{\circ} 1$, Belo Horizonte.

Hirschman, A.O. (1958), The Strategy of Economic Development, New Haven, Yale University Press.

Hongyi, W. (2009), "The Analysis of Input-Output in Hospital and the Practical Application in Medicine Health Domain", documento presentado en la Decimoséptima Conferencia Internacional sobre Técnicas de Insumo-Producto.

IBGE (Instituto Brasileño de Geografía y Estadística) (2008), Economia da saúde: uma perspectiva macroeconômica 20002005, Río de Janeiro

(s/f), "Matriz de insumo-produto (2000 e 2005)" [en línea] http://www.ibge.gov.br/.

Imori, D. y J.J.M. Guilhoto (2007), "Estrutura produtiva brasileira e emissão de CO2", Anais do V Encontro da Associação Brasileira de Estudos Regionais e Urbanos, Recife.

Luna, E. (2002), "A emergência das doenças emergentes e as doenças infecciosas emergentes e reemergentes no Brasil", Revista Brasileira de Epidemiologia, vol. 5, $\mathrm{N}^{\circ}$, Associação Brasileira de Saúde Coletiva.
Marinho, A., S.S. Cardoso y V.V. Almeida (2009), "Brasil e ocDE: avaliação da eficiência em sistemas de saúde", Texto para Discussão, $\mathrm{N}^{\circ}$ 1370, Instituto de Investigación Económica Aplicada (IPEA).

Mattos, R.S. y otros (2008), "Integração de modelos econométricos e de insumo produto para previsões de longo prazo da demanda de energia no Brasil", Estudos Econômicos, vol. 38, São Paulo, Universidad de São Paulo.

Miller, R. y P. Blair (2009), Input-Output Analysis: Foundations and Extensions, Nueva Jersey, Prentice-Hall.

Moreira, G. (2007), "Politicas sociais, desigualdades pessoais e regionais da renda no Brasil: uma analise de insumo-produto", Escuela Superior de Agricultura "Luiz de Queiroz".

OCDE (Organización para la Cooperación y el Desarrollo Económicos) (2010), “OECD Health Data 2010: Statistics and Indicators" [en línea] http://www.oecd.org.br.

oMs (Organización Mundial de la Salud) (2010), "Indicadores sanitarios mundiales", Estadísticas sanitarias mundiales 2010, Ginebra [en línea] http://www.who.int/whosis/whostat/ ES_WHS10_Full.pdf?ua=1.

(2009), Estadísticas sanitarias mundiales 2009, Ginebra [en línea] http://www.who.int/gho/publications/world health statistics/2009/es/.

(2001), Macroeconomics and Health: Investing in Health for Economic Development. Report of the Commission on Macroeconomics and Health, Ginebra, Organización Mundial de la Salud (oms).

Perobelli, F.S., R.S. Mattos y W.R. Faria (2007), "Interações energéticas entre o Estado de Minas Gerais e o restante do Brasil: uma análise inter-regional de insumo-produto", Economia Aplicada, vol. 11, $\mathrm{N}^{\circ}$ 1, São Paulo, Universidad de São Paulo.

Rasmussen, P.N. (1952), Studies in Inter-Sectoral Relations, Amsterdam, North-Holland.

Ribeiro, M.B. (2009), "Eficiência do gasto público na América Latina: uma análise comparativa a partir do modelo semi-paramétrico com estimativa em dois estágios", serie Gestión Pública, Nº67 (LC/L.2883-P), Santiago de Chile, Comisión Económica para América Latina y el Caribe (CEPAL). Publicación de las Naciones Unidas, $\mathrm{N}^{\circ}$ de venta: P.08.II.G.28.

(2008), "Desempenho e eficiência do gasto público: uma análise comparativa entre o Brasil e um conjunto de países da América Latina", Texto para Discussão, N 1368 , Brasilia, Instituto de Investigación Económica Aplicada (IPEA).

Ribeiro, M.B. y W. Rodrigues Jr. (2006), "Eficiência do gasto público na América Latina", Boletim de Desenvolvimento Fiscal, $\mathrm{N}^{\circ} 3$, Instituto de Investigación Económica Aplicada (IPEA).

Robine, J.M., I. Romieu y E. Cambois (1999), "Indicadores de la esperanza de salud", Bulletin of the World Health Organization. Recopilación de artículos, $\mathrm{N}^{\circ} 1$, Ginebra, Organización Mundial de la Salud (oms)

Rodrigues, R.L. y J.J.M. Guilhoto (2004), "Estrutura produtiva, relações intersetoriais e cooperativas agropecuárias no Paraná em 1980 e 1985", Revista de Economia e Sociologia Rural, vol. 42, $\mathrm{N}^{\circ} 2$, Sociedad Brasileña de Economía y Sociología Rural.

Schramm, J.N.A. y otros (2004), "Transição epidemiológica e o estudo de carga de doença no Brasil", Ciência \& Saúde Coletiva, vol. 9 , № 4, Asociación Brasileña de Salud Colectiva.

Sesso Filho, U.A. y otros (2006), "Interações sinérgicas e transbordamento do efeito multiplicador de produção das grandes regiões do Brasil", Economia Aplicada, vol. 10, $\mathrm{N}^{\circ} 2$, São Paulo, Universidad de São Paulo.

Sonis, M. y G.J.D. Hewings (1992), "Coefficient changes in inputoutput models: theory and applications", Economic Systems Research, vol. 4, $\mathrm{N}^{\circ} 2$. 
Terra, L.P. y B.L. Queiroz (2010), "Qualidade de vida: qual é a esperança de vida feliz no Brasil?", Anais do XVII Encontro Nacional de Estudos Populacionais, Caxambu, Minas Gerais.
Toyoshima, S. y M.J. Ferreira (2002), "Encadeamentos do setor de transportes na economia brasileira", Planejamento e Políticas Públicas, $\mathrm{N}^{\circ} 25$, Instituto de Investigación Económica Aplicada (IPEA). 